\title{
Whole genome sequencing in the diagnosis of primary ciliary dyskinesia
}

\author{
Gabrielle Wheway ${ }^{1,2^{*}+}$ (D) N. Simon Thomas ${ }^{1,3+}$, Mary Carroll ${ }^{5,6}$, Janice Coles ${ }^{5,6}$, Regan Doherty, ${ }^{5,6,7}$, \\ Genomics England Research Consortium, Patricia Goggin 5,6,7, Ben Green ${ }^{8}$, Amanda Harris ${ }^{5,6}$, David Hunt ${ }^{4}$, \\ Claire L. Jackson ${ }^{5,6}$, Jenny Lord ${ }^{1,2}$, Vito Mennella ${ }^{2,5,6}$, James Thompson ${ }^{5,6}$, WoolfT. Walker ${ }^{5,6}$ and Jane S. Lucas , $^{5,6^{*}}$
}

\begin{abstract}
Background: It is estimated that 1-13\% of cases of bronchiectasis in adults globally are attributable to primary ciliary dyskinesia (PCD) but many adult patients with bronchiectasis have not been investigated for PCD. PCD is a disorder caused by mutations in genes required for motile cilium structure or function, resulting in impaired mucociliary clearance. Symptoms appear in infancy but diagnosis is often late or missed, often due to the lack of a "gold standard" diagnostic tool and non-specific symptoms. Mutations in $>50$ genes account for around $70 \%$ of cases, with additional genes, and non-coding, synonymous, missense changes or structural variants (SVs) in known genes presumed to account for the missing heritability.
\end{abstract}

Methods: UK patients with no identified genetic confirmation for the cause of their PCD or bronchiectasis were eligible for whole genome sequencing (WGS) in the Genomics England Ltd 100,000 Genomes Project. 21 PCD probands and 52 non-cystic fibrosis (CF) bronchiectasis probands were recruited in Wessex Genome Medicine Centre (GMC). We carried out analysis of single nucleotide variants (SNVs) and SVs in all families recruited in Wessex GMC.

Results: $16 / 21$ probands in the PCD cohort received confirmed $(n=9)$, probable $(n=4)$ or possible $(n=3)$ diagnosis from WGS, although 13/16 of these could have been picked up by current standard of care gene panel testing. In the other cases, SVs were identified which were missed by panel testing. We identified variants in novel PCD candidate genes (IFT140 and PLK4) in 2 probands in the PCD cohort. 3/52 probands in the non-CF bronchiectasis cohort received a confirmed $(n=2)$ or possible $(n=1)$ diagnosis of PCD. We identified variants in novel PCD candidate genes (CFAP53 and CEP164) in 2 further probands in the non-CF bronchiectasis cohort.

Conclusions: Genetic testing is an important component of diagnosing PCD, especially in cases of atypical disease history. WGS is effective in cases where prior gene panel testing has found no variants or only heterozygous variants. In these cases it may detect SVS and is a powerful tool for novel gene discovery.

Keywords: Primary ciliary dyskinesia, Non-CF bronchiectasis, Whole genome sequencing, Diagnosis, Gene discovery

*Correspondence: g.wheway@soton.ac.uk; jlucas1@soton.ac.uk ${ }^{\dagger} G$ abrielle Wheway and N. Simon Thomas have contributed equally.

${ }^{1}$ School of Human Development and Health, Faculty of Medicine, University of Southampton, Southampton, UK

${ }^{5}$ School of Clinical and Experimental Sciences, Faculty of Medicine, University of Southampton, Southampton, UK

Full list of author information is available at the end of the article

\section{Background}

Bronchiectasis is a disorder of persistent or recurrent bronchial infection, chronic cough, and purulent sputum production, associated with permanent airway dilatation. It is the end-point of various diseases and pathological mechanisms, and diagnosing bronchiectasis should therefore be the starting point to finding the underlying cause. Globally, severe lung infections such 
as tuberculosis, whooping cough, or measles can damage the airways at the time of infection, predisposing to a cycle of inflammation, structural airway damage and further infection culminating in irreversible bronchiectasis. In most Western populations, genetic conditions including cystic fibrosis (CF), primary ciliary dyskinesia (PCD) and primary immunodeficiency disorders are common causes of bronchiectasis. CF, caused by mutations in the CFTR gene is the most common genetic cause $(\approx 1: 2500$ European Caucasians), and is usually picked up before bronchiectasis is established by new-born screening where it is available. PCD is less common $(\approx 1: 10,000$ Caucasians, although higher in consanguineous populations eg 1:2200 in British Asians [1]), and despite having symptoms from infancy is often diagnosed in late childhood or adulthood [2]. The reasons for missed or late diagnoses are multi-factorial including no "gold standard" diagnostic tool, insufficient specialist diagnostic centres and clinicians' lack of awareness about PCD [3]. PCD symptoms of daily wet cough, persistent rhinorrhoea, sinusitis and serous otitis media are non-specific. Patients with dextrocardia, which is rare in the general population but affects $50 \%$ of people with PCD, are diagnosed earlier than those with normal organ situs [2].

A combination of investigations is usually required to make a diagnosis of PCD $[4,5]$. Whilst transmission electron microscopy (TEM) or genetics testing can confirm the diagnosis, each will miss $20-30 \%$ of patients, and other tests are therefore important $[4,6]$. Whilst molecular genetic investigations play an essential role in the diagnosis of PCD, genetic results can be difficult to interpret without confirming the phenotype by TEM, immunofluorescent labelling of ciliary proteins and/ or highspeed video microscopy of ciliary beat pattern [79]. It is important to note that when all test results are 'normal', PCD cannot be ruled out conclusively albeit high-unlikely.

Mutations that cause PCD have been reported in $>50$ genes [10]. These account for around $70 \%$ of cases, with additional genes, and non-coding, synonymous, missense changes or structural variants (SVs) in known genes presumed to account for the missing heritability. Many adult patients with non-CF bronchiectasis have not been investigated for PCD although it is estimated that $1-13 \%$ of cases of bronchiectasis in adults worldwide are attributable to PCD [11]. This wide range is due to different screening tools and diagnostic tests being used to diagnose PCD, and so it is difficult to assess the true rate of PCD in the adult bronchiectasis community. In the UK, patients suspected to have PCD can be sent for gene panel testing on a panel of known PCD disease genes alongside functional and structural tests of clia. This original panel consisted of 19 genes and later 29 genes
(Additional file 1: Table S1). Whole genome sequencing (WGS) provides a second line opportunity for identification of non-coding changes and SVs in known PCD genes which may be missed by standard gene panel testing, and also provides opportunities for novel disease gene identification. UK patients with no genetic confirmation for the cause of their PCD or bronchiectasis were eligible for WGS in the UK 100,000 Genomes Project. The 100,000 Genomes Project, coordinated by Genomics England Ltd, aimed to deliver clinical benefits to patients by providing genetic diagnoses for people with cancer and rare diseases, with an additional and critically important spin off of providing a large amount of data for research. Furthermore this hybrid clinical and research project aimed to integrate WGS into the UK National Health Service (NHS), and to create the National Genomic Medicine Service [12, 13]. Recruitment to the project was coordinated across 13 Genome Medicine Centres (GMCs). Wessex GMC, covering Southampton University Hospital NHS Foundation Trust, a specialist PCD diagnostic centre, and Queen Alexandra Hospital, Portsmouth was the main recruiting GMC for non-CF bronchiectasis and the second largest recruiter of PCD probands in the 100,000 Genomes Project. We carried out clinical review of all variants returned from $\mathrm{PCD}$ and non-CF bronchiectasis patients by the 100,000 Genomes Project. This involved clinical variant classification following guidelines from the American College of Medical Genetics (ACMG) and the Association for Molecular Pathology (AMP) [14] and the current ACGS guidelines [15] to determine whether a variant was pathogenic, likely pathogenic, a variant of uncertain clinical significance, benign or likely benign. Genome sequence files, alignment files, variant call files and tiering files were also made available to approved researchers in a secure online research environment and we carried out comprehensive independent research analysis of these files for patients recruited in Wessex GMC, with an aim to identify pathogenic variants missed by the automated Genomics England Ltd tiering process, and uplift genetic diagnosis in these cohorts.

\section{Methods}

Patient selection and recruitment to the 100,000 Genomes Project

Recruitment to the 100,000 Genomes Project was initially restricted to patients who had undergone prior genetic diagnostic testing and had not received a genetic diagnosis, although later in the project this was relaxed to include patients who had not undergone previous genetic testing. The PCD cohort $(n=42$, of which probands $\mathrm{n}=21$ ) comprised patients from the PCD Adult and Paediatric Centre at University Hospital Southampton 
(UHS) who had no genetic diagnosis of PCD, and their first-degree relatives. 9/21 probands had no prior genetic testing. $1 / 21$ probands had previously had targeted sequencing of DNAH11, 7/21 had been tested against a gene panel which included 19 known PCD genes (Additional file 1: Table S1), and 4/21 had previously been tested against a gene panel which included 29 known PCD genes (Additional file 1: Table S1). Of the 12 patients with prior testing, 9 had variants identified but these were either heterozygous $(2 / 12)$ or did not confirm a genetic diagnosis (because of 1 or more variants were variants of uncertain clinical significance (VUS)). These patients were recruited for WGS as part of 100,000 Genomes Project. The non-CF bronchiectasis cohort $(n=69$, of which probands $n=52)$ included patients under adult pulmonology care at UHS and Portsmouth Hospitals NHS Trust. Informed consent was obtained for participation in the 100,000 Genomes Project, and where appropriate for PCD diagnostic testing (National Research Ethics Service South Central (A) Committee 07/Q1702/109 and University of Southampton Faculty of Medicine Ethics Committee ERGO\#53,155). Clinical characteristics were recorded using Human Phenotype Ontology (HPO) terms and blood taken for DNA extraction.

\section{Diagnostic testing in Southampton PCD diagnostic service} PCD diagnostic testing was undertaken for all participants in the PCD cohort prior to recruitment to 100,000 Genomes Project. Participants in the non-CF bronchiectasis cohort were invited for PCD functional testing and biomedical imaging if the WGS found variants in PCDrelated genes. A detailed clinical history was recorded using a proforma. PCD diagnostic testing included nasal nitric oxide (nNO), high-speed video microscopy (HSVM) analysis [8], transmission electron microscopy (TEM) and immunofluorescence (IF) microscopy imaging, as previously described [8, 16-19]. In brief, $\mathrm{nNO}$ was measured by chemiluminescence analyser (Ecomedics CLD 88 Exhalyzer) during exhalation against resistance with sampling at $0.33 \mathrm{l} / \mathrm{min}$ and a cut-off of $77 \mathrm{~nL} / \mathrm{min}$. High-speed video analysis was conducted in a $37{ }^{\circ} \mathrm{C}$ heated environmental chamber; images were digitally recorded using a high-speed camera at a rate of 500 frames per second (fps) and reviewed at reduced frame rates (30-60 fps) for analysis of ciliary beat pattern (CBP) and ciliary beat frequency (CBF). To differentiate primary from secondary dyskinesia samples were reanalysed following in vitro differentiation of basal epithelial cells and ciliation in an air-liquid interface culture [20]. 100-300 cilia were imaged by Hitachi H7000 electron microscope in transverse section for qualitative and quantitative assessment of axonemal structure (minimum magnification 60,000×) [19]. Immunofluorescent confocal imaging of targeted PCD related proteins used a Leica SP8 confocal microscope to identify mislocalised or absent proteins [21]. Antibodies used included RPGR Sigma HPA001593 (1:200), Alpha tubulin clone DM1A Sigma T9026 (1:500), Alexafluor 488 Invitrogen A21121 (1:2500) and Alexafluor 594 Invitrogen A11012 (1:2500). Confocal immunofluorescence images of nasal brushings and ALI cultures were taken on a Leica TCSSP8 laser scanning confocal system with true spectral detection attached to a Leica DMI8 inverted microscope frame and using Leica LAS-X v3.5.7 acquisition software. All images were taken using a Leica HC PL APO CS2 63x / 1.3 N.A. glycerol immersion lens as single Z slices at a visually-optimised focal plane. Imaging parameters were $1024 \times 1024$ pixel sampling $(X Y), 600 \mathrm{~Hz}$ scan speed, 4 line averaging and $-1 \%$ offset for background correction and $3 \times$ optical zoom (by restricting angular deflection of the scanning mirrors), providing nyquist sampling at a $80 \mathrm{~nm}$ pixel size (XY). Sequential imaging (DAPI + AF594/AF488) was used to avoid spectral bleed though between channels. The photomultiplier gain for the RPGR antibody was set to a constant level for all the images. No post processing of images was performed.

\section{Whole genome sequencing and data analysis}

A detailed description of the 100,000 Genomes Project protocol can be found at https://www.genomicsen gland.co.uk/wp-content/uploads/2017/03/GenomicEng landProtocol_151117-v4-Wales.pdf. In brief, DNA was extracted and stored in a central bioresource, PCR-free libraries prepared and sequenced using Illumina shortread technology, sequence data aligned to the human reference genome, followed by variant calling and annotation. Genomics England Ltd performed variant filtering through an automated analysis pipeline to identify potentially diagnostic small nucleotide variants (SNVs) and structural variants (SVs), which were "tiered" according to likelihood of being the pathogenic cause of disease if in clinically relevant genes (Tables 1,2 ) [22] and consistent with the observed pattern of inheritance [12]. Tier 1 and 2 SNVs (Fig. 1) and all SVs $>10 \mathrm{~kb}$ (ie variants most likely to be clinically relevant) were returned to GMCs for clinical review and classification of variants following ACMG/AMP guidelines [14] and the current ACGS guidelines [15] to determine whether they were pathogenic, likely pathogenic, a variant of uncertain clinical significance, likely benign or benign. These were then reported back to recruiting clinicians and patients.

In parallel, all anonymised sequence data, small nucleotide variant and structural variant files, HPO data and tiering data for each participant was made available for interrogation by approved researchers through the secure 
Table 1 Green genes on the primary ciliary disorders PanelApp virtual gene panel, considered diagnostic for PCD in the clinical analysis pipeline of the 100,000 Genomes Project

\begin{tabular}{|c|c|c|}
\hline Gene Name & Model_Of_Inheritance & OMIM number \\
\hline ARMC4 & Autosomal recessive & 615451 \\
\hline C21orf59 & Autosomal recessive & 615500 \\
\hline CCDC103 & Autosomal recessive & 614679 \\
\hline CCDC114 & Autosomal recessive & 615067 \\
\hline CCDC151 & Autosomal recessive & 616037 \\
\hline CCDC39 & Autosomal recessive & 613807 \\
\hline CCDC40 & Autosomal recessive & 613808 \\
\hline CCDC65 & Autosomal recessive & 615504 \\
\hline CCNO & Autosomal recessive & 615872 \\
\hline DNAAF1 & Autosomal recessive & 613193 \\
\hline DNAAF2 & Autosomal recessive & 612518 \\
\hline DNAAF3 & Autosomal recessive & 606763 \\
\hline DNAAF4 & Autosomal recessive & 127700 \\
\hline DNAAF5 & Autosomal recessive & 614874 \\
\hline DNAH11 & Autosomal recessive & 611884 \\
\hline DNAH5 & Autosomal recessive & 608644 \\
\hline DNAl1 & Autosomal recessive & 244400 \\
\hline DNAI2 & Autosomal recessive & 612444 \\
\hline DNAL1 & Autosomal recessive & 614017 \\
\hline$D R C 1$ & Autosomal recessive & 615294 \\
\hline GAS8 & Autosomal recessive & 616726 \\
\hline HYDIN & Autosomal recessive & 608647 \\
\hline$L R R C 6$ & Autosomal recessive & 614935 \\
\hline MCIDAS & Autosomal recessive & 618695 \\
\hline PIH1D3 & X-linked recessive & 300991 \\
\hline$R P G R$ & X-linked recessive & 300455 \\
\hline RSPH1 & Autosomal recessive & 615481 \\
\hline RSPH3 & Autosomal recessive & 616481 \\
\hline RSPH4A & Autosomal recessive & 612649 \\
\hline RSPH9 & Autosomal recessive & 612650 \\
\hline SPAG1 & Autosomal recessive & 615505 \\
\hline ZMYND10 & Autosomal recessive & 615444 \\
\hline
\end{tabular}

Gene name, pattern of inheritance and OMIM ID are provided for each gene. This panel was originally 31 genes at the start of the project; PIH1D3 was added towards the end of the project. All PCD patients had this panel applied. Some but not all non-CF bronchiectasis patients had this panel applied online Research Environment within the Genomics England Data Embassy. PCD and non-CF bronchiectasis research analysis was conducted on this data in the research environment. Further details can be found in Additional file 2.

\section{Clinical review and classification of variants}

We reviewed all tier 1 and tier 2 variants (Fig. 1) and $\mathrm{SVs}>10 \mathrm{~kb}$ using ACMG/AMP guidelines for clinical variant interpretation [14] and the current ACGS guidelines [15]. Where appropriate, variants were classified at a genomics MDT which included reviewing PCD diagnostic results where available. Although PCD is genetically heterogeneous, review of the PCD clinical diagnostic testing data (nNO, HSVM, TEM and IF) enabled the PP4 line of evidence to be applied to some specific cases (a supporting level of evidence that a variant is pathogenic can be applied when the patient's phenotype, in this case cellular phenotype, is highly specific for the gene of interest). Where a SV was identified, the corresponding sequence data were manually interrogated. Sequence and coverage data were also manually inspected to verify the presence of variants identified SNVs were confirmed by Sanger sequencing or other parallel testing. SVs were confirmed by PCR amplification of the deletion breakpoint and Sanger sequencing of the PCR product.

Where tiered variants did not provide a diagnosis, we proceeded to use custom Python scripts to extract heterozygous SNVs in known PCD genes, and heterozygous and homozygous SNVs in candidate ciliopathy genes [23, $24]$, and $S V s<10 \mathrm{~kb}$ in all genes of interest using WGS data for all PCD and non-CF bronchiectasis probands recruited in Wessex GMC.

This analysis was carried out under approved Genomics England research project RR185, with ethical approval from University of Southampton Faculty of Medicine Ethics Committee (ERGO\#54,400).

\section{Results}

PCD cohort participants

The 100,000 Genomes Project recruited 118 PCD patients nationally (274 individuals when including

Table 2 Green genes on the non-CF bronchiectasis PanelApp virtual gene panel, considered diagnostic for non-CF bronchiectasis and CF in the clinical analysis pipeline of the 100,000 Genomes Project

\begin{tabular}{lll}
\hline Gene Name & Model_Of_Inheritance & OMIM number \\
\hline CFTR & Autosomal recessive & 219700 \\
PIK3CD & Autosomal dominant (not imprinted) & 615513 \\
SCNN1A & Autosomal dominant (imprinted status unknown) & 613021 \\
SCNN1B & Autosomal dominant, autosomal recessive & 211400 \\
SCNN1G & Autosomal dominant (not imprinted) & 613071
\end{tabular}

Gene name, pattern of inheritance and OMIM ID are provided for each gene 


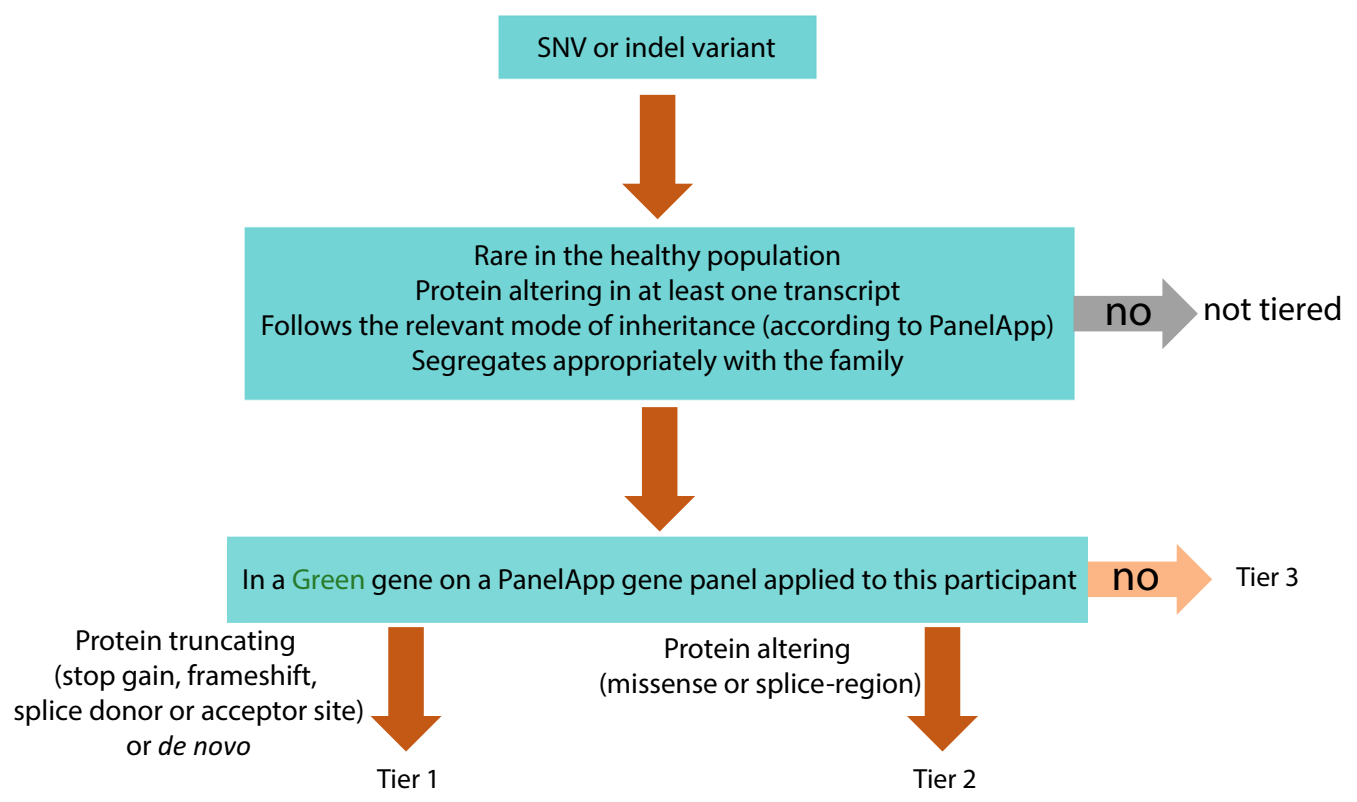

Fig. 1 Genomics England Ltd (Genomics England Ltd) variant filtering and tiering strategy for single nucleotide variants (SNVs) or small insertions or deletions. Tier 1 and tier 2 variants were returned to recruiting Genome Medicine Centres (GMC) for variant review and classification following American College of Medical Genetics guidelines. Tier 3 variants were available for review in the research environment. Primary ciliary disorders PanelApp gene panel can be found in Table 1, non-CF bronchiectasis PanelApp gene panel can be found in Table 2

probands and family members). 21 PCD probands were recruited in Wessex GMC (41 participants including family members) including 9 singletons, 4 duos and 8 trios. We first describe the results returned in the clinical tiering analysis, and then the results of our independent research analysis.

\section{Results of SNV analysis in PCD cohort}

Tier 1 or tier 2 variants were found in $12 / 21$ patients. ACMG variant classification confirmed a diagnosis of PCD in 6 cases (biallelic pathogenic/likely pathogenic variants) (Table 3; cases 1-6). The causative genes were DRC1, DNAI1, DNAH11 $(\times 2)$ and DNAH5 $(\times 2)$. Of the 6 confirmed cases, 4 had not undergone prior testing but the variants were all identifiable by standard panel testing. A diagnosis was now possible in the 2 cases that had undergone prior testing because either additional cases were available in the literature or parental testing demonstrated that the variants were in trans (allowing the PM3 line of evidence to be applied); WGS did not identify any additional variants.

A further 3 cases received a probable diagnosis (biallelic pathogenic or likely pathogenic variant and VUS in a known PCD gene) (Table 3; cases 10-12). The variants were in DNAI1 and DNAH11 $(\times 2)$. All had undergone prior targeted $D N A H 11$ or gene panel testing and received the same findings. Re-analysis of the VUS did not result in reclassification, and so the diagnosis remains as probable but not confirmed. WGS did not provide useful additional information in these cases but confirmed findings from gene panel testing.

A further 3 cases received a possible diagnosis (biallelic VUS(s) in a known PCD gene) (Table 3; 15-17). Two of these had undergone prior gene panel testing and received the same result. The genes were DNAAF1, DNAH5 and DNAH11. Again, re-analysis of the VUS did not result in reclassification and so the diagnosis remains as possible but not confirmed. One case had not undergone prior testing, and so received a new result, but this could have been obtained through gene panel testing rather than WGS.

\section{Results of SV analysis in PCD cohort}

$\mathrm{SV}>10 \mathrm{~kb}$ analysis identified a heterozygous pathogenic $\mathrm{SV}$ of around $27 \mathrm{~kb}$ in DNAH5, deleting exons 8-16 (inclusive) in one family. Manual inspection of DNAH5 sequence data identified a stop gain in trans with the SV providing an additional confirmed diagnosis (Table 3; case 7). This heterozygous stop gain variant was not tiered (due to being heterozygous) and so this diagnosis could have been missed by the Genomics England Ltd pipeline. This participant had undergone prior gene panel testing which identified the heterozygous DNAH5 stop gain variant but not the SV. In this case WGS was useful in providing confirmed genetic diagnosis in this individual which was not possible with gene panel testing. 
Table 3 Genetic testing results from panel testing, 100,000 Genomes Project whole genome sequencing, clinical history and PCD diagnostic test results for each proband $1-21$ in the Wessex PCD cohort

\begin{tabular}{|c|c|c|c|c|c|c|c|c|c|c|}
\hline \multirow{2}{*}{$\begin{array}{l}\text { Ca } \\
\text { se } \\
\text { ID }\end{array}$} & \multicolumn{4}{|c|}{ Genetics } & \multicolumn{6}{|c|}{ Clinical information } \\
\hline & Gene & Allele 1 [Classification] & $\begin{array}{c}\text { Allele 2 } \\
\text { [Classification] }\end{array}$ & $\begin{array}{l}\text { Diagnos } \\
\text { is }\end{array}$ & $\begin{array}{l}\text { Clinical } \\
\text { History }\end{array}$ & \begin{tabular}{|c} 
nNO \\
(nL/m \\
in)
\end{tabular} & $\begin{array}{c}\text { HSV- } \\
\text { brushing }\end{array}$ & $\begin{array}{c}\text { HSV- } \\
\text { postALI }\end{array}$ & TEM & IF \\
\hline 1 & $\begin{array}{l}\text { DNAH } \\
{ }_{11}\end{array}$ & $\begin{array}{l}\text { DNAH1I GRCh38 7:21619178C }>\mathrm{T} \text {, } \\
\text { NM_001277115.2:.43333C } \mathrm{T} \text {, p.R1445X. } \\
\text { Maternal. [Pathogenic] }\end{array}$ & \begin{tabular}{|l} 
DNAHII GRCh38 \\
7:21787442G $>$ C, \\
NM_001277115.2c. 97 \\
83G $>$ C, p.E3261D. \\
Paternal. [VUS]. \\
GRCh38 \\
7:21901009G $>$ C, \\
NM_001277115.2c. 13 \\
306G $>$ C, p.A4436P. \\
Paternal. [Likely \\
Pathogenic] \\
\end{tabular} & $\begin{array}{c}\text { Confirm } \\
\text { ed }\end{array}$ & $\begin{array}{l}\text { SI, NRDS, } \\
\text { daily wet } \\
\text { cough, } \\
\text { persistent } \\
\text { rhinitis, no } \\
\text { ear issues }\end{array}$ & $\begin{array}{l}\text { Too } \\
\text { young }\end{array}$ & $\begin{array}{l}\text { Static and } \\
\text { twitching }\end{array}$ & Not done & Normal & $\begin{array}{l}\text { Not } \\
\text { done }\end{array}$ \\
\hline 2 & $\begin{array}{c}\text { DNAH } \\
5\end{array}$ & $\begin{array}{l}\text { DNAH5 GRCh38 5:13753289GA }>\mathrm{G} \\
\text { NM_001369.3:c.10815del p.D3605delfs1. } \\
\text { Paternal [Pathogenic] }\end{array}$ & $\begin{array}{l}\text { DNAH5 GRCh38 } \\
\text { 5:13753490G }>\text { A } \\
\text { NM_001369.3:c.10615 } \\
\text { C }>\text { T, p. R3539C. } \\
\text { Maternal. [Likely } \\
\text { pathogenic] }\end{array}$ & $\begin{array}{c}\text { Confirm } \\
\text { ed }\end{array}$ & $\begin{array}{l}\text { SI, no } \\
\text { NRDS, } \\
\text { wet cough } \\
\text { and } \\
\text { rhinitiis } \\
\text { since } \\
\text { infancy, } \\
\text { no ear } \\
\text { issues } \\
\end{array}$ & 16 & \begin{tabular}{|l} 
Static and \\
occasional \\
twitching
\end{tabular} & Not done & ODA & $\begin{array}{l}\text { Not } \\
\text { done }\end{array}$ \\
\hline 3 & DNAII & $\begin{array}{l}\text { DNAII GRCh38 9:34512199G }>\mathrm{A} \\
\text { NM_012144.4:c. } 1401+1 \mathrm{G}>\mathrm{A} \text { [Likely } \\
\text { Pathogenic] }\end{array}$ & Homozygous & $\begin{array}{c}\text { Confirm } \\
\text { ed }\end{array}$ & $\begin{array}{l}\text { SI, } \\
\text { persistent } \\
\text { wet cough } \\
\text { since } \\
\text { infancy, } \\
\text { rhinosinus } \\
\text { itus, } \\
\end{array}$ & 7 & Static & Not done & ODA & $\begin{array}{l}\text { Not } \\
\text { done }\end{array}$ \\
\hline 4 & $\begin{array}{l}\text { DNAH } \\
11\end{array}$ & $\begin{array}{l}\text { DNAHII GRCh38 7:21900087G }>\mathrm{T} \text {, } \\
\text { NM_001277115.2c:.13270G }>\mathrm{T} \text {, p.E4424X } \\
\text { [Likely Pathogenic] }\end{array}$ & $\begin{array}{l}\text { DNAH1] GRCh38 } \\
\text { 7:21901076C }>\mathrm{T}, \\
\text { NM_01277115.2:c. } 13 \\
\text { 373 C }>\text { T, p.P4458L } \\
\text { [Likely Pathogenic] }\end{array}$ & $\begin{array}{c}\text { Confirm } \\
\text { ed }\end{array}$ & $\begin{array}{l}\text { Normal } \\
\text { situs, } \\
\text { cough } \\
\text { since } \\
\text { infancy, } \\
\text { bronchiect } \\
\text { asis, } \\
\text { sinusitis, } \\
\text { hearing } \\
\text { loss. } \\
\end{array}$ & 6 & $\begin{array}{l}\text { Hyperfreq } \\
\text { uent } \\
\text { twitching }\end{array}$ & $\begin{array}{l}\text { Mostly } \\
\text { static }\end{array}$ & Normal & $\begin{array}{l}\text { Not } \\
\text { done }\end{array}$ \\
\hline 5 & DNAII & $\begin{array}{l}\text { DNAII GRCh38 9:34459054G>GT, } \\
\text { NM_012144.4:c.48+1dup [Pathogenic] }\end{array}$ & Homozygous & $\begin{array}{c}\text { Confirm } \\
\text { ed }\end{array}$ & \begin{tabular}{|l} 
Normal \\
situs, wet \\
cough \\
since \\
infancy, \\
severe \\
bronchiect \\
asis, \\
rhinosinus \\
itis. \\
\end{tabular} & $\begin{array}{l}\text { Not } \\
\text { done }\end{array}$ & Not done & Not done & ODA & $\begin{array}{l}\text { Not } \\
\text { done }\end{array}$ \\
\hline 6 & $D R C l$ & $\begin{array}{l}\text { DRCl GRCh38 2:26421396C }>\mathrm{T} ; \\
\text { NM_145038.5c.352C } \mathrm{T} ; \mathrm{p} \text {. Q118X } \\
\text { [Likely Pathogenic] }\end{array}$ & Homozygous & $\begin{array}{c}\text { Confirm } \\
\text { ed }\end{array}$ & \begin{tabular}{|l|} 
Normal \\
situs,, \\
persistent \\
wet cough \\
since \\
childhood, \\
bronchiect \\
asis, \\
rhino- \\
sinusitis, \\
glue ear \\
\end{tabular} & 99 & $\begin{array}{l}\text { Double } \\
\text { beat } \\
\text { pattern }\end{array}$ & \begin{tabular}{|l|} 
Static \\
and \\
twitching
\end{tabular} & \begin{tabular}{|l} 
MTD \\
with \\
normal \\
inner \\
arms
\end{tabular} & $\begin{array}{l}\text { Not } \\
\text { done }\end{array}$ \\
\hline 7 & $\begin{array}{c}\text { DNAH } \\
5\end{array}$ & $\begin{array}{l}\text { DNAH5 GRCh38 } \\
\text { 5:13891193 } 13918248 \text { del, } \\
\text { NM 001369.3c. } 975 \text { 2431del. Paternal. } \\
\text { [Pathogenic] }\end{array}$ & $\begin{array}{l}\text { DNAH5 GRCh38 } \\
\text { 5:13701316dup } \\
\text { NM_001369.3.c.13459 } \\
\text { ins; p. .144878. } \\
\text { Maternal [Pathogenic] }\end{array}$ & $\begin{array}{c}\text { Confirm } \\
\text { ed }\end{array}$ & \begin{tabular}{|l} 
Normal \\
situs, \\
NRDS, \\
persistent \\
wet \\
cough, \\
rrinitis \\
and ear \\
infections
\end{tabular} & 18 & $\begin{array}{l}\text { Static and } \\
\text { dyskinetic }\end{array}$ & Not done & ODA & $\begin{array}{l}\text { Not } \\
\text { done }\end{array}$ \\
\hline 8 & $\begin{array}{c}D N A A \\
F 4\end{array}$ & $\begin{array}{l}\text { DNAAF4 GRCh38 } \\
15: 55437707155440622 \text { del, } \\
\text { NM_130810.4:c.784_893del. [Pathogenic] }\end{array}$ & $\begin{array}{l}\text { DNAAF4 (GRCh38 } \\
\text { 15:55435013A>T; } \\
\text { NM_130810.4:c.939T> } \\
\text { A; p.Y313X) } \\
\text { [Pathogenic] }\end{array}$ & $\begin{array}{c}\text { Confirm } \\
\text { ed }\end{array}$ & \begin{tabular}{|l|} 
Normal \\
situs, \\
NRDS, \\
persitent \\
wet cough \\
and nasal \\
congestion \\
since early \\
childhood, \\
glue ear, \\
iimmotile \\
sperm \\
\end{tabular} & 13 & Static & Static & $\begin{array}{l}\text { ODA+I } \\
\text { DA }\end{array}$ & $\begin{array}{l}\text { Not } \\
\text { done }\end{array}$ \\
\hline 9 & $\begin{array}{c}D N A H \\
11\end{array}$ & $\begin{array}{l}\text { DNAHII GRCh38 } \\
\text { 7:21606634_21616124del, } \\
\text { NM_001277115.2: c.3766_4011. Not } \\
\text { paternal, no maternal sample [Likely } \\
\text { Pathogenic] }\end{array}$ & $\begin{array}{l}\text { DNAH1I GRCh38 } \\
\text { 7:21900000C }>\mathrm{T}, \\
\text { NM 001277115.2:c. } 13 \\
\text { 183C }>\text { T, p.R4395X. } \\
\text { Paternal. [Pathogenic] }\end{array}$ & $\begin{array}{l}\text { Confirm } \\
\text { ed }\end{array}$ & \begin{tabular}{|l|} 
SI, no \\
NRDS, \\
persistant \\
productive \\
cough, \\
rhino- \\
sinusitus, \\
glue ear. \\
\end{tabular} & 30 & $\begin{array}{l}\text { Static and } \\
\text { twitching }\end{array}$ & Not done & $\begin{array}{l}\text { Equivo } \\
\text { cal }\end{array}$ & $\begin{array}{l}\text { Not } \\
\text { done }\end{array}$ \\
\hline 10 & $\begin{array}{c}\text { DNAH } \\
I I\end{array}$ & $\begin{array}{l}\text { DNAHII GRCh38 7:21616208G }>\mathrm{C} \\
\text { NM_001277115.2:c.4012-1G }>\mathrm{C} \text {. Maternal } \\
\text { [Pathogenic] }\end{array}$ & $\begin{array}{l}\text { DNAH1I GRCh38 } \\
\text { 7:21894771G }>\mathrm{A}, \\
\text { NM_ 00127715.2:c.12 } \\
\text { 899 }>\text { G }>\text { A, p. R 4300H. } \\
\text { Paternal. [VUS] }\end{array}$ & Probable & \begin{tabular}{|l|} 
SIT, few \\
pulmonary \\
symtoms, \\
persistant \\
rhinitis, no \\
ear issues.
\end{tabular} & 62 & $\begin{array}{l}\text { Dyskinesti } \\
\text { c and } \\
\text { hyperfrequ } \\
\text { ent }(n=3 \\
\text { occasions) }\end{array}$ & \begin{tabular}{|l|} 
Static \\
$(\mathrm{n}=3$ \\
occasions \\
)
\end{tabular} & Normal & $\begin{array}{l}\text { DNAH1 } \\
1 \\
\text { staining } \\
\text { present }\end{array}$ \\
\hline 11 & DNAII & $\begin{array}{l}\text { DNAIII GRCh38 9:345144468G >A, } \\
\text { NM_0121214.4:c. } 16444 \mathrm{~A}>\mathrm{A} \text {, p.W548X. } \\
\text { Paternal. [Pathogenic] }\end{array}$ & $\begin{array}{l}\text { DNAII GRCh38 } \\
\text { 9:34513157T>C, } \\
\text { NM_012144.4.c.1535T } \\
\text { >C, p.F512S. Maternal. } \\
\text { [VUS] }\end{array}$ & Probable & \begin{tabular}{|l} 
Normal \\
situs, \\
NRDS, \\
persistent \\
wet \\
cough, \\
rhinitis, \\
recurrent \\
glue ear. \\
\end{tabular} & 13 & Static & $\begin{array}{l}\text { Unsucces } \\
\text { sful }\end{array}$ & ODA & $\begin{array}{l}\text { Not } \\
\text { done }\end{array}$ \\
\hline
\end{tabular}


Table 3 (continued)

\begin{tabular}{|c|c|c|c|c|c|c|c|c|c|c|}
\hline 12 & $\begin{array}{c}D N A H \\
I I\end{array}$ & $\begin{array}{l}\text { DNAH1I GRCh38 7:21749702C }>\text { T, } \\
\text { NM_001277115.2:c.8698C }>\text { T, p.R2900X. } \\
\text { Paternal [Pathogenic] }\end{array}$ & $\begin{array}{l}\text { DNAH11 GRCh38 } \\
\text { 7:21738827C }>\text { T, } \\
\text { NM_001277115.2c. } 77 \\
\text { 72C }>\text { T, p.P2591L. } \\
\text { Maternal. [VUS] }\end{array}$ & Probable & $\begin{array}{l}\text { Normal } \\
\text { situs, no } \\
\text { NRDS, } \\
\text { presistet } \\
\text { wet cough } \\
\text { and nasal } \\
\text { congestion } \\
\text { since } \\
\text { infancy, } \\
\text { glue ear } \\
\end{array}$ & 5 & $\begin{array}{l}\text { Twitching } \\
\text { and } \\
\text { hyperkinet } \\
\text { ic, some } \\
\text { static }\end{array}$ & Not done & Nomal & $\begin{array}{l}\text { Not } \\
\text { done }\end{array}$ \\
\hline 13 & HYDIN & $\begin{array}{l}\text { HYDIN GRCh38 16:71051500- } \\
\text { 71061418del, } \\
\text { NM_001270974.2:c.2377_2529. Maternal. } \\
\text { [VUS] }\end{array}$ & $\begin{array}{l}\text { HYDIN GRCh38 } \\
\text { 16:71162577G }>\mathrm{A}, \\
\text { NM_130810.4c.670C }> \\
\text { T, p.R224X. Paternal } \\
\text { [Pathogenic] }\end{array}$ & Probable & \begin{tabular}{|l} 
Normal \\
situs, \\
NRDS \\
with lobar \\
collapse, \\
persistent \\
wet \\
cough, \\
persistent \\
rhinitis, no \\
ear issues.
\end{tabular} & 9 & $\begin{array}{l}\text { Dyskinetic } \\
\text { reduced } \\
\text { amplitude. }\end{array}$ & $\begin{array}{l}\text { Dyskineti } \\
\text { c, some } \\
\text { static } \\
\text { with } \\
\text { residual } \\
\text { moveme } \\
\text { nt }\end{array}$ & $\begin{array}{l}\text { Normal } \\
\mathrm{n}=2\end{array}$ & $\begin{array}{l}\text { SPEF2 } \\
\text { very } \\
\text { weak/ab } \\
\text { sent }\end{array}$ \\
\hline 14 & $\begin{array}{c}C C D C \\
114\end{array}$ & $\begin{array}{l}\text { CCDCI14 GRCh38 19:48311552C }>\mathrm{T} \text {, } \\
\text { NM 001364171.2: } 2.597+1 \text { (no parent } \\
\text { info) [Pathogenic] }\end{array}$ & $\begin{array}{l}\text { CCDC114 GRCh38 } \\
\text { 19:48307171T >G, } \\
\text { NM 001364171.2: } 59 \\
\text { 8-8-848 A }>\text { C (no parent } \\
\text { info) [VUS] }\end{array}$ & $\begin{array}{c}\text { Unresol } \\
\text { ved. }\end{array}$ & $\begin{array}{l}\text { Normal } \\
\text { situs, } \\
\text { NRDS, } \\
\text { persistent } \\
\text { wet } \\
\text { cough, } \\
\text { bronchiect } \\
\text { asis, } \\
\text { rhinosinus } \\
\text { itis, glue } \\
\text { ear. }\end{array}$ & 20 & Static & $\begin{array}{l}\text { static and } \\
\text { occasion } \\
\text { al } \\
\text { sluggish } \\
\text { moveme } \\
\text { nt }\end{array}$ & normal & $\begin{array}{l}\text { Not } \\
\text { done }\end{array}$ \\
\hline 15 & $\begin{array}{c}D N A H \\
I I\end{array}$ & $\begin{array}{l}\text { DNAHII GRCh38 7:21570283G }>\mathrm{A} \text {, } \\
\text { NM_001277115.2:c.1409G }>\mathrm{A}, \mathrm{p} . \mathrm{R} 470 \mathrm{H} \\
\text { [VUS] GRCh38 7:21735664A }>\mathrm{C} \\
\text { NM_001277115.2:c.4333C }>\mathrm{T} \text {, p.R1445X } \\
\text { [VUS] }\end{array}$ & Homozygous & Possible & \begin{tabular}{|l|} 
SI, no \\
NRDS, \\
neonatal \\
rhinitis, \\
persistent \\
wet cough \\
and \\
rhinitis \\
since \\
infancy \\
\end{tabular} & 12 & $\begin{array}{l}\text { Dyskinetic } \\
\text { and } \\
\text { uncoordin } \\
\text { ated; } 4 \\
\text { occasions }\end{array}$ & $\begin{array}{l}\text { Static; } 3 \\
\text { occasions }\end{array}$ & Normal & $\begin{array}{l}\text { DNAH1 } \\
1 \\
\text { present }\end{array}$ \\
\hline 16 & $\begin{array}{c}D N A H \\
5\end{array}$ & $\begin{array}{l}\text { DNAH5 GRCh38 5:13829650G>A, } \\
\text { NM _001369.3:c.6304C }>\text { T, p.R2102C. } \\
\text { Maternal. [VUS] }\end{array}$ & $\begin{array}{l}\text { DNAH5 GRCh38 } \\
\text { 5:13841886A>G, } \\
\text { NM_001369.3.c.5290T } \\
>\text { C, p.S1764P. } \\
\text { Paternal. [VUS] } \\
\text { DNAH5 GRCh38 } \\
\text { 5:13901505A>G, } \\
\text { NM_001369.3:c.1799T } \\
>\text { C, p.I600T. Paternal. } \\
\text { [VUS] }\end{array}$ & Possible & $\begin{array}{l}\text { SI NRDS } \\
\text { daily wet } \\
\text { cough, } \\
\text { nose OK, } \\
\text { mild } \\
\text { hearing } \\
\text { loss. }\end{array}$ & 8 & Static & Not done & ODA & $\begin{array}{l}\text { Not } \\
\text { doen }\end{array}$ \\
\hline 17 & $\begin{array}{c}D N A A \\
F I\end{array}$ & $\begin{array}{l}\text { DNAAFI GRCH38 16:84155575C }>\mathrm{G} \text {; } \\
\text { NM_178452.6:-575-8C }>\mathrm{A} \text { SpliceAI delta } \\
\text { score for acceptor loss (at }+8 \text { ) } 0.91 \text {, delta } \\
\text { score for acceptor gain (at }+1) 0.66 \text {. } \\
\text { Maternal. [VUS] }\end{array}$ & $\begin{array}{l}\text { DNAAFI GRCh38 } \\
\text { 16:84155691C }>\text { T; } \\
\text { NM_178452.6:c.683C } \\
\text { T; p.S228L). Paternal } \\
\text { [VUS] }\end{array}$ & Possible & $\begin{array}{l}\text { Normal } \\
\text { situs, } \\
\text { NRDS, } \\
\text { persistent } \\
\text { wet } \\
\text { cough, } \\
\text { hhinitis, } \\
\text { glue ear, }\end{array}$ & 16 & Static & Not done & $\begin{array}{l}\text { ODA } \\
\text { and } \\
\text { IDA }\end{array}$ & $\begin{array}{l}\text { Not } \\
\text { done }\end{array}$ \\
\hline 18 & IFTI40 & $\begin{array}{l}\text { IFT140 missense GRCh38 } \\
\text { 16:1562105C }>\text { G; NM 014714.4 } \\
\text { c.2079G }>\text { C; p.L693F (maternal) }\end{array}$ & $\begin{array}{l}\text { IFT140 missense } \\
\text { GRCh38 } \\
\text { 16:1584407T>C; } \\
\text { NM_014714.4 } \\
\text { c.1169A }>\text { G; p.K390R } \\
\text { (not in mother) }\end{array}$ & $\begin{array}{c}\text { Candida } \\
\text { te }\end{array}$ & $\begin{array}{l}\text { Normal } \\
\text { situs, } \\
\text { NRDS, } \\
\text { persisent } \\
\text { wet } \\
\text { cough, } \\
\text { glue ear }\end{array}$ & 8 & $\begin{array}{l}\text { Twitching } \\
\text { and } \\
\text { hyperfrequ } \\
\text { ent }\end{array}$ & Not done & normal & $\begin{array}{l}\text { Not } \\
\text { done }\end{array}$ \\
\hline 19 & PLK4 & $\begin{array}{l}\text { PLK4 GRCh38 } \\
\text { 4:127890202TAAAACCAATCAGACAG } \\
\text { >T; } \\
\text { NM } 014264.5: c .1796 \text { 1812delAAAACCA } \\
\text { ATCAGACAG; p.L599delfs6X (maternal) }\end{array}$ & $\begin{array}{l}\text { PLK4 GRCH38 } \\
\text { 4:127881931G }>\text { C } \\
\text { NM_014264.5:c.126+5 } \\
\text { G }>\text { C SpliceAI delta } \\
\text { score for donor loss (at } \\
-5 \text { ) } 0.47 \text { (paternal) }\end{array}$ & $\begin{array}{c}\text { Candida } \\
\text { te }\end{array}$ & $\begin{array}{l}\text { Normal } \\
\text { situs, no } \\
\text { NRDS, } \\
\text { persistent } \\
\text { wet cough } \\
\text { and } \\
\text { rhinitis } \\
\text { since early } \\
\text { infancy, } \\
\text { glue ear }\end{array}$ & 12 & $\begin{array}{l}\text { Stiff, } \\
\text { uncoordin } \\
\text { ated }\end{array}$ & Not done & Normal & $\begin{array}{l}\text { Not } \\
\text { done }\end{array}$ \\
\hline 20 & N/A & No findings & No findings & $\begin{array}{l}\text { Not } \\
\text { PCD }\end{array}$ & $\begin{array}{l}\text { PCD } \\
\text { history not } \\
\text { taken }\end{array}$ & 108 & $\begin{array}{l}\text { Several } \\
\text { samples } \\
\text { all with } \\
\text { health } \\
\text { issues but } \\
\text { dyskinesia } \\
\text {,although } \\
\text { not typical } \\
\text { of PCD }\end{array}$ & $\begin{array}{l}\text { Not } \\
\text { suggestiv } \\
\text { e of PCD }\end{array}$ & Normal & $\begin{array}{l}\text { Not } \\
\text { done }\end{array}$ \\
\hline 21 & $\mathrm{~N} / \mathrm{A}$ & No findings & No findings & $\begin{array}{l}\text { Not } \\
\text { PCD }\end{array}$ & 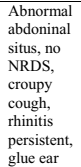 & 180 & $\begin{array}{l}\text { Normal } \\
\text { (2014) }\end{array}$ & Not done & $\begin{array}{l}\text { Not } \\
\text { done }\end{array}$ & $\begin{array}{l}\text { Not } \\
\text { done }\end{array}$ \\
\hline
\end{tabular}

\section{ACMG variant classification for each variant is given in bold}

The table provides case ID, gene and variant details (with GRCh38 genomic coordinates) and classification (following ACMG guidelines) and final diagnosis. Clinical information provided includes clinical history, nasal nitric oxide levels ( $\mathrm{nNO}$ ( $\mathrm{nL} / \mathrm{min})$ ), high-speed video (HSV) microscopy analysis from fresh nasal brushings and afterculture of these cells at air-liquid interface (ALI) transmission electron microscopy (TEM) and immunofluorescence (IF) microscopy findings. SI = Situs inversus, $\mathrm{NRDS}=$ neonatal respiratory distress syndrome, ODA = outer dynein arm defect, IDA = inner dynein arm defect, MTD = Microtubular disorganisation 


\begin{abstract}
(See figure on next page.)
Fig. 2 Structural variants in known PCD genes identified in unsolved patients in the Wessex PCD cohort. IGV screenshot showing heterozygous $3.5 \mathrm{~kb}$ SV in DNAAF4 deleting exon 7 in case 8 in Table 3. This was found in trans with a stop gain variant in DNAAF4, providing a confirmed diagnosis for this patient. a IGV screenshot showing heterozygous 9.5 kb SV in DNAH11 deleting exon 20 and 21 in case 9 in Table 3. This results in an in-frame deletion of amino acids 1256-1337 containing a coiled-coil domain which may take part in protein-protein interactions. Multiple alignment of DNAH11 protein sequence across multiple species shows that this region of the protein is well conserved and likely to be functionally important, so that the deletion would be deleterious to protein function. The ACMG classification was likely pathogenic. This was found in trans with a pathogenic stop gain variant in DNAH11, providing a confirmed diagnosis for this patient. b IGV screenshot showing heterozygous $\sim 9.8 \mathrm{~kb}$ SV in HYDIN deleting exon 18 in case 13, affected sibling and mother but not father. This results in an in-frame deletion of amino acids 793-843 of the 5121 amino acid protein. Multiple alignment of HYDIN protein sequence across multiple species shows that this region of the protein is conserved and likely to be functionally important, but the ACMG classification was variant of uncertain significance because we couldn't show that the deleted interval was critical to protein function. This was found in trans with a stop gain variant in HYDIN (also found in affected sibling and father), providing a probable diagnosis for this patient and their affected sibling. c Immunofluorescence confocal image of SPEF2 (red), ciliary axoneme marker alpha tubulin (green) and DAPI, overlaid with phase contrast image of single multiciliated cells from the nasal epithelium of health volunteer (left) and PCD case 13, who has a $9.8 \mathrm{k}$ deletion in HYDIN in trans with a stop gain variant in HYDIN. HYDIN is required for trafficking of SPEF2 into the cilia, and so absence of SPEF2 in the cilia of this patient is supportive of the genetic findings in this patient
\end{abstract}

In summary, $13 / 21$ participants received confirmed, probable or possible diagnostic results from the initial clinical analysis of tiered data and SVsGenomics England Ltd. The causative genes were DNAAF1, DRC1, DNAI1 $(\times 3), D N A H 11(\times 5)$ and DNAH5 $(\times 3)$. These were new findings in $6 / 13$ cases and in $7 / 13$ cases confirmed results from prior panel testing.

$8 / 21$ participants did not receive a result from the initial analysis of tiered variants.

From the 8 negative cases, we proceeded to extract and study heterozygous SNVs in known PCD genes, and found 4 heterozygous potentially pathogenic stop gain or splice site variants (Table 3 ; cases $8-9+13-14$ ) in DNAAF4, DNAH11, HYDIN and CCDC114. One of these had undergone prior gene panel testing that had identified the single heterozygous SNV in DNAAF4. We then visually analysed the alignment files of these participants in IGV to identify any SVs. This identified that 3 of the cases had biallelic SNVs and SVs in PCD genes which were not picked up by the tiering pipeline, as they were $<10 \mathrm{~kb})$ :

- $\quad$ stop gain $+\sim 3.5 \mathrm{~kb} \mathrm{SV}$ in DNAAF4 deleting exon 7 (Table 3; case 8, Fig. 2a)

- stop gain + 9.5 kb SV in DNAH11 deleting exon 20 and 21 (Table 3; case 9, Fig. 2b)

- stop gain $+\sim 9.8 \mathrm{~kb} \mathrm{SV}$ in HYDIN deleting exon 18 (Table 3; case 13, Fig. 2c)

The DNAAF4, DNAH11 and HYDIN SNVs and SVs were found to segregate appropriately in the family and were all classified as pathogenic following ACMG guidelines, except the HYDIN SV which was classified as VUS. This provides 2 further confirmed diagnoses and 1 probable diagnosis. We were satisfied that HYDIN was the causative gene by observing absence of SPEF2 in the motile cilia of the proband, shown by IF confocal imaging (Fig. 2d). HYDIN is required for trafficking of SPEF2 into the cilia, and so absence of SPEF2 in the cilia of this patient is supportive of the genetic findings in this patient [25]. However, due to issues around poor mapping quality caused by HYDIN pseudogene, HYDIN2, we plan to carry out additional RNA investigations to further corroborate these genetic findings. We are confident that the stop gain is in HYDIN as sequence reads show it is in cis with an intronic SNV that is divergent between HYDIN and HYDIN2 but wish to confirm that the SV is specific to HYDIN. The two DNAH11 findings were novel, this patient did not undergo prior testing. The two HYDIN findings were novel, despite this patient having undergone 29 gene panel testing previously. In these cases WGS was extremely useful in providing confirmed genetic diagnosis in these individuals. It is unlikely that these SVs could have been detected by the small custom PCD gene panel testing.

No SVs were found in CCDC114, but we did find a novel deep intronic variant in CCDC114 which is absent from gnomAD and may affect splicing, although this is not predicted by SpliceAI [26]. Segregation analysis was not possible in this case, as the proband was recruited as a singleton. Furthermore, this individual had normal ciliary ultrastructure by TEM, but outer dynein arm defects would be expected in a CCDC114 patient. The official ACMG classification of this intronic variant is variant of uncertain significance (VUS), and with the second pathogenic SNV in CCDC114 this provides a probable diagnosis following ACMG guidelines. However, ERS PCD Diagnostic guidelines say "Genetic diagnosis has to be consistent with the clinical and TEM/IF/HSVA phenotype, or diagnosis reconsidered if the picture is inconsistent" and so, on balance, we class this as unresolved, with additional work (RNA studies) required to further investigate this case. 
a

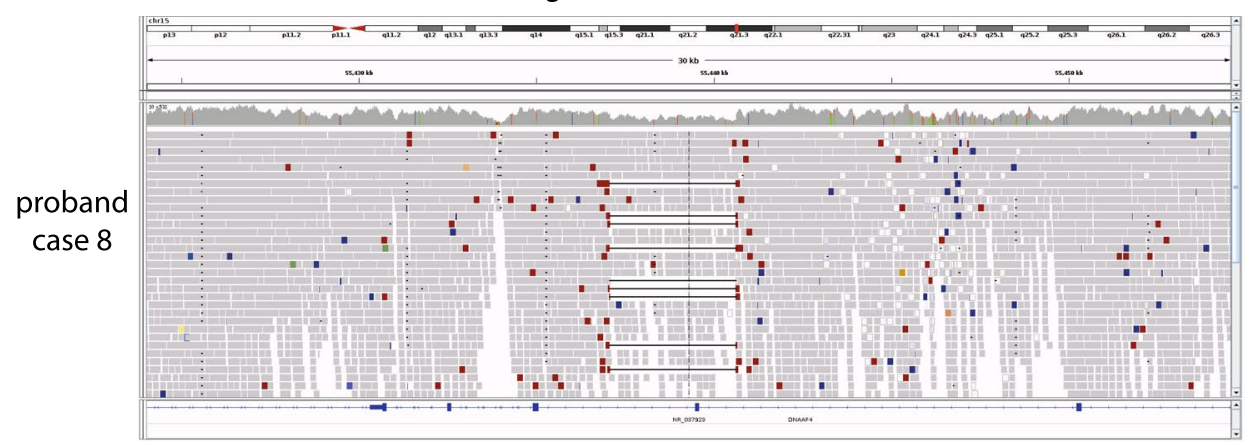

b

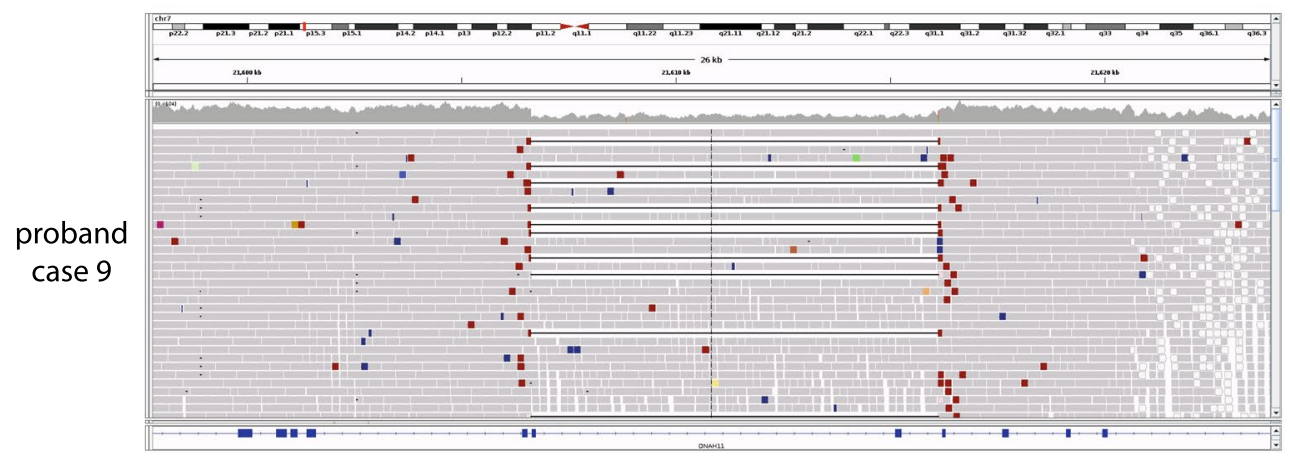

C

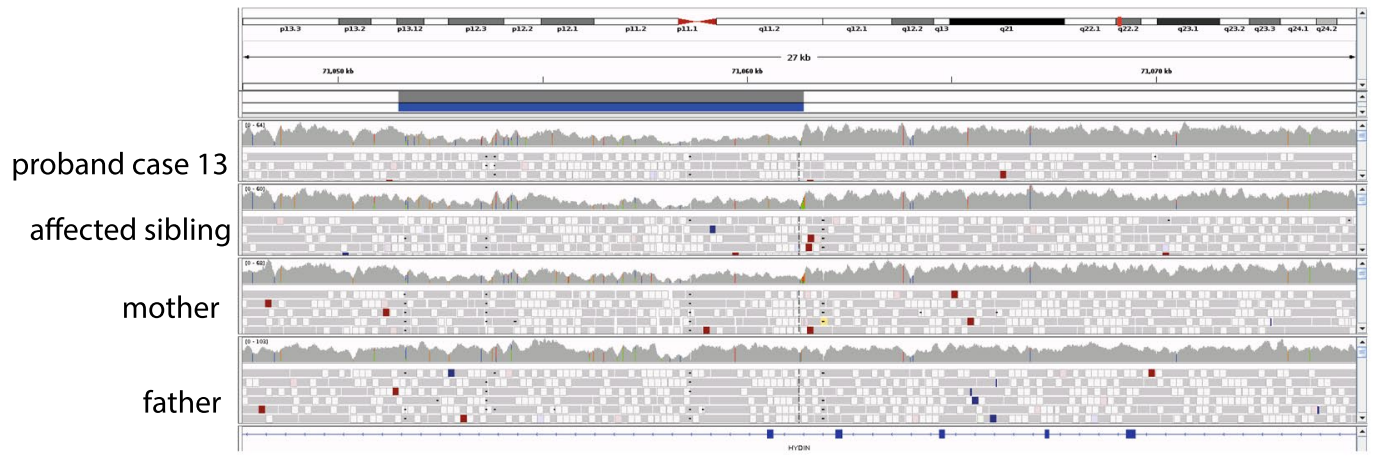

d

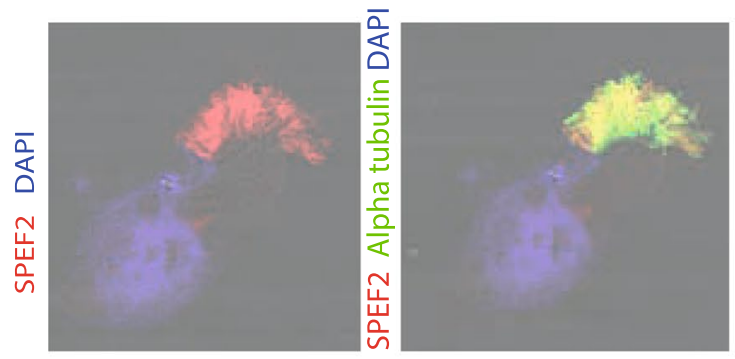

Healthy volunteer

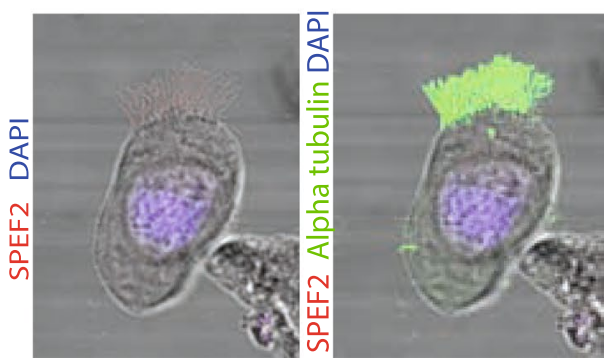

HYDIN proband

Fig. 2 (See legend on previous page.) 
This left 4/21 additional unsolved PCD cases, 2 of whom had undergone prior gene panel testing. In these cases we studied tier 3 variants, applying a functional candidate gene approach by using an updated version of the Syscilia Gold Standard list of known cilia genes [23] and CiliaCarta list of candidate cilia genes [24]. This identified biallelic variants in two cilia or centrosomal genes which have been linked to other OMIM diseases but not PCD (Table 3; cases 18+19): IFT140, which has been reported in isolated retinitis pigmentosa (RP) [27, 28] and syndromic RP [29] and several short rib polydactyly syndromes [30-32] and PLK4 which has been associated with microcephaly and chorioretinopathy [33]. The IFT140 variants are both missense variants; a rare p.(Leu693Phe) which is predicted by PolyPhen to be tolerated and by SIFT to be benign, and a rare p.(Lys390Arg) which is predicted by PolyPhen to be tolerated and by SIFT to be benign. The PLK4 variants are a frameshift loss-of-function and a -5 splice variant which is predicted to cause loss of the splice donor site, with a SpliceAI donor loss delta score of 0.47 (delta scores $>0.5$ are reliable predictors of a biological effect on splicing). In order to validate $P L K 4$ as the causative gene, RNA analysis will need to be carried out. In these patients, WGS will have proved invaluable if our further research work can confirm diagnoses in these cases. In the remaining two cases no candidate variants were identified and re-review of the clinical phenotype information indicated that historical diagnostics were not suggestive of PCD, and these patients were inappropriately recruited within the PCD disease category. In these cases WGS analysis was useful in confirming that these patients had no genetic cause of PCD, although this could have been concluded through re-review of their clinical notes.

\section{Summary of WGS results from patients recruited in PCD cohort}

In total 16 participants received confirmed, probable or possible genetic diagnosis from all analyses (Fig. 3). In all cases the diagnostic phenotype, including cellular and ciliary phenotype, was consistent with the genotype. One additional case had genetic findings sufficient for a probable diagnosis (ie Pathogenic variant+VUS) following ACMG classification, but this was at odds with TEM findings; the variants were in CCDC114, where ODA defects would be expected on TEM but were not seen. Following ERS PCD diagnostic guidelines this case remains unresolved. 13 of the diagnoses were provided by Genomics England Ltd tiering analysis and 3 were obtained through further research analysis of the WGS data. 3/16 patients had homozygous SNVs, 13/16 patients were compound heterozygous and in 4 of these patients one of the variants was a SV. Only 1 of these SVs was reported by Genomics England Ltd, as it was $>10 \mathrm{~kb}$. The other 3 were found manually and were all $<10 \mathrm{~kb}$. Furthermore, research analysis identified variants in candidate PCD genes (IFT140 and PLK4) in 2 cases. Thorough genetic analysis and diagnostic imaging found no evidence of PCD in 2 cases. In the 16 cases which received a diagnosis, the causative genes were: $D N A A F 1$, DNAAF4, DRC1, DNAI1 (×3), DNAH11 (×6), DNAH5 $(\times 3)$ and $H Y D I N$ (Fig. 4). As variants in DRC1 have not been reported in many patients, we report the clinical features of the $D R C 1$ patient here.

\section{DRC1 case study}

A 34-year-old man from a non-consanguineous background presented with a persistent wet cough since childhood, sino-rhinitis, glue ear and bronchiectasis. He had a PICADAR score [34] of $4 / 14$ which is below the level recommended for referral for diagnostic testing. Nasal nitric oxide (nNO) levels were within the normal range (CLD 88 Exhalyzer; exhalation against resistance; $109 \mathrm{nl} / \mathrm{min}$ ). High speed video microscopy (HSVM) of nasal epithelial cells showed uncoordinated cilia with an intermittent double beat 'tick' and limited movement. The beat pattern was persistently abnormal on four different occasions, and also following culture at air liquid interface; according to international guidelines this is not diagnostic but makes a positive diagnosis highly likely [4]. TEM showed 20\% of cilia displayed microtubular disorganisation (MTD) which in itself is insufficient to diagnose PCD, but might suggest mutations in $C C D C 65$, $D R C 1$ or GAS8 encoding nexin-dynein regulatory complex proteins [19]. Genetic testing as part of a research programme in 2013 (not standard of care testing, therefore not recorded in Table 3 ) had failed to identify a causative gene, and six years later this project finally provided a definitive diagnosis of PCD caused by homozygous stop gain mutation in DRC1 (CCDC164); a gene described to cause PCD in 2013 [35].

Therefore, although functional and imaging tests were abnormal, they did not confirm a definite diagnosis of PCD. Using high speed video and TEM the abnormalities were subtle, likely to be missed by non-specialists. Indeed the literature sometimes describes high speed video and TEM as normal in patients with mutations in $D R C 1$. In this patient $\mathrm{nNO}$ levels were normal, although they have previously been reported as abnormally low in patients with mutations in DRC1. We were not using immunofluorescent analyses at the time the patient was diagnosed, but would anticipate absence of GAS8 localisation in the cilia, as DRC1 and GAS8 are both components of the nexin-dynein regulatory complex (N-DRC) 
PCD cohort

$\mathrm{n}=21$ probands

GEL analysis

A: SNVs in primary ciliary gene panel genes (tier $1 / 2$ variants)

B: SVs $>10 \mathrm{~kb}$ in primary ciliary gene panel genes (tier A variants) (applied to 8 families only)

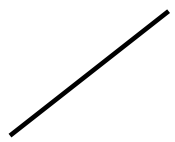

$8 / 21$ probands unresolved

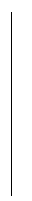

Research analysis

A: biallelic SNVs in cilia candidate genes (from tier 3 variants)

B. monoallelic SNVs in primary ciliary gene panel genes (untiered)

C: SVs $<10 \mathrm{~kb}$ in primary ciliary gene panel genes (untiered)

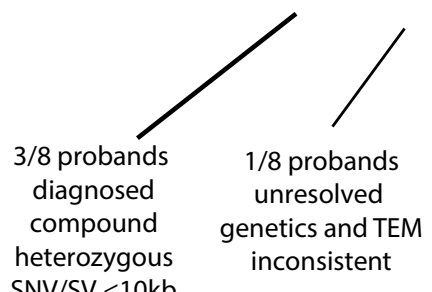

SNV/SV $<10 \mathrm{~kb}$
13/21 probands diagnosed

11 compound heterozygous SNVs 3 homozygous SNVs

1 compound heterozygous SNV/SV $>10 \mathrm{~kb}$

Fig. 3 Flowchart showing clinical and research analyses carried out and all results for Wessex PCD cohort

in the cilium and mutations in DRC1 cause N-DRC defects which can be observed as absence of GAS8 localisation from the cilium [36]. This case highlights the important role of genetic testing in variants where functional and imaging tests might be normal or equivocal, and also the need to revisit inconclusive cases as new PCD-causing genes are described.

\section{Non-CF bronchiectasis cohort participants}

52 non-CF bronchiectasis probands were recruited in Wessex GMC (69 participants including family members), including 44 singletons. Between 2 and 9 different PanelApp panels were applied to these cases, with an average of 2.5 panels. None had prior genetic testing for PCD. We first describe the results of the analysis carried out by Genomics

(See figure on next page.)

Fig. 4 Causative genes in PCD cases recruited as PCD or recruited as non-CF bronchiectasis. a Pie chart chowing proportion of patients recruited into PCD disease category with causative variants in different known and candidate PCD genes. $\mathbf{b}$ Pie chart chowing proportion of patients recruited into non-CF disease category with causative variants in different known and candidate PCD genes. $\mathbf{c}$ Pie chart chowing proportion of patients recruited into PCD and non-CF disease categories with causative variants in different known and candidate PCD genes 


\section{a}

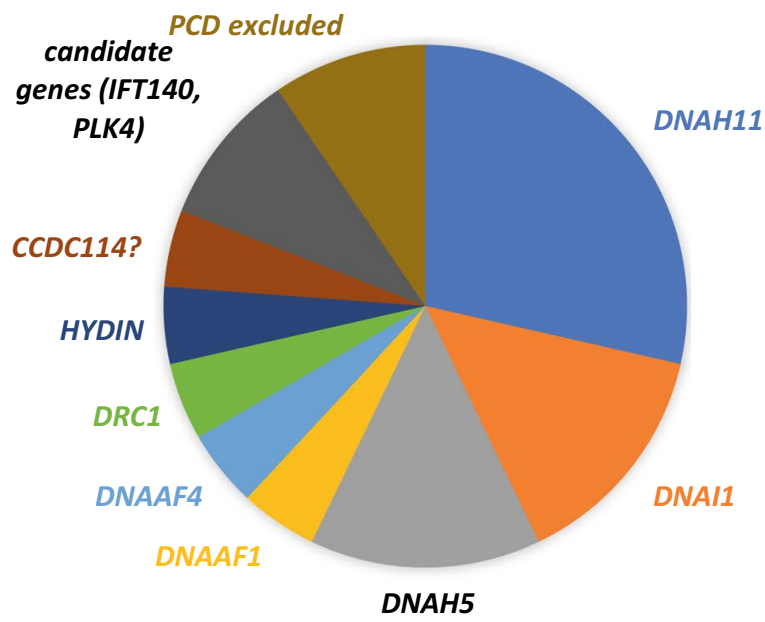

recruited as $P C D$

b

candidate genes (CEP164,

CFAP53)

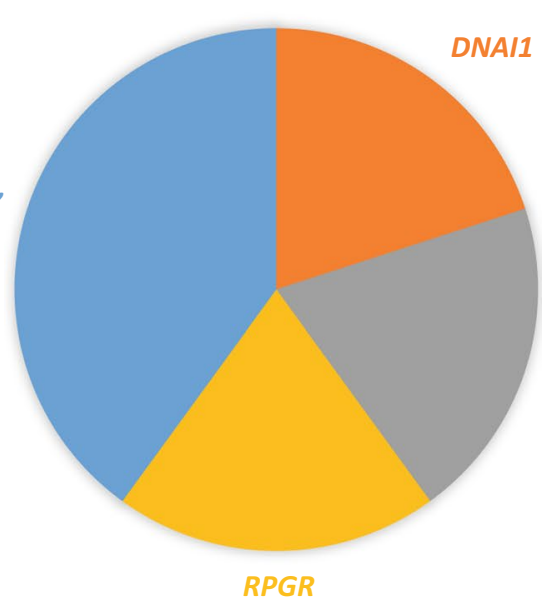

recruited as non-CF

bronchiectasis

DNAH5

C

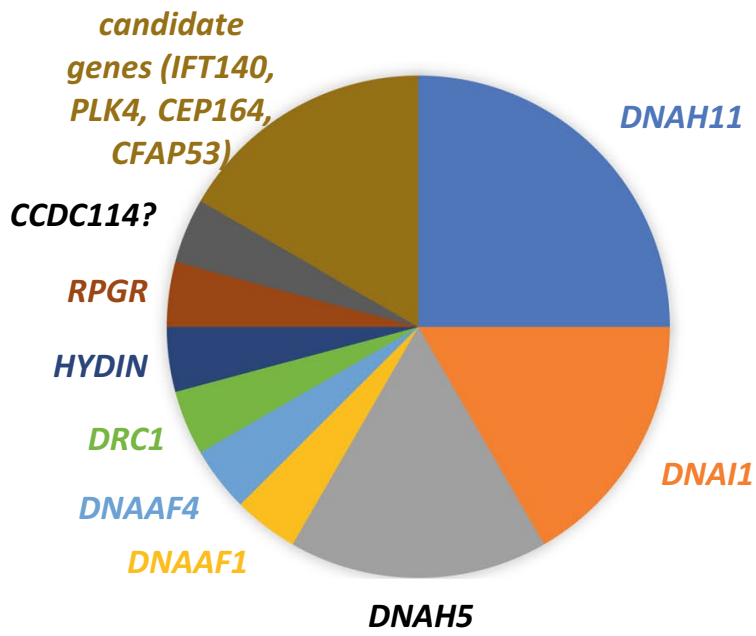

all confirmed PCD

Fig. 4 (See legend on previous page.) 
England Ltd and reviewed in the GMC, and then describe the results of our independent research analysis.

\section{Results of SNV analysis in non-CF bronchiectasis cohort}

In the initial analyses the PCD virtual gene panel was applied to only 37 families. 3 individuals had tier 1 or 2 variants in a $P C D$ gene. After variant classification following ACMG guidelines, diagnosis of PCD was confirmed in $2 / 3$ of these individuals (causative genes $D N A 1$ and DNAH5) and the diagnosis of PCD was possible in $1 / 3$ individuals (causative gene RPGR) (Table 4, Fig. 5). Although $R P G R$ is formally a VUS, IF result suggests it is the cause of the patient's PCD (Fig. 6).

The DNAI1 patient had a typical clinical history (situs inversus, neonatal respiratory distress, daily wet cough, perennial rhinitis, bronchiectasis and glue ear). This patient was referred for PCD diagnostics, which was typical for a person with DNAH1 disease (low nNO, static cilia on HSVM and TEM showed outer dynein arm defects). Similarly the DNAH5 patient had a classical clinical history and diagnostic findings (low nNO, static cilia before and after culture at air-liquid interface (ALI), TEM showed outer dynein arm defects and IF showed absence of DNAH5 from the cilium).

Whilst review of the RPGR patient's clinical history was typical for PCD, an ophthalmologist confirmed the patient's description of no eye disease, despite the association of $R P G R$ variants with $\mathrm{X}$-linked retinitis pigmentosa. This patient subsequently underwent PCD diagnostics, which showed a low nNO, abnormal HSVM before and after culture at air-liquid interface (ALI), dyskinetic throughout. TEM showed normal cilia ultrastructure, but IF imaging showed absence of RPGR from the cilium (Fig. 6). The patient was male and the variant was a hemizygous RPGR missense p.(His201Arg), absent from gnomAD, predicted by Polyphen to be probably damaging (score 0.996 ) and by SIFT to be deleterious (score 1).

These cases demonstrate how even in large bronchiectasis clinics, cases of PCD can be missed, and in these cases WGS can be invaluable for genetic diagnosis. The $R P G R$ case further confirms the contribution of $R P G R$ variants in PCD and provides some evidence that the RPGR PanelApp rating should be reviewed as it has been down-graded in the current PCD panel.

In the remaining unsolved non-CF bronchiectasis cases we did not find any untiered SNVs or SVs in PCD genes so we applied a functional candidate gene approach by using an updated version of the Syscilia Gold Standard list of known cilia genes [23] and CiliaCarta list of candidate cilia genes [24]. This identified biallelic variants in two cilia or centrosomal genes which have been linked to other OMIM diseases but not PCD (Table 4, Fig. 5).
One individual had biallelic CFAP53 stop gain variants which segregated within the family. CFAP53 variants have been associated with visceral heterotaxy type 6 in patients $[37,38]$ but the gene has not been previously described in PCD. Whilst this protein is present along the axoneme of cilia on human nasal respiratory epithelial cells, motility defects have not been described in the respiratory cilia in patients with CFAP53 mutations [37]. However, recent work in mice has shown that tracheal and ependymal cilia show an altered beat pattern in Cfap53 mutant mice [39]. In these mice the $9+0$ nodal cilia (required for left-right patterning) are completely immotile, but the $9+2$ motile cilia have subtle defects in the beat pattern. The respiratory phenotype of these mice was not described. Clinical review of our patient showed situs inversus, no NRDS, recurrent chest infections which started in adulthood, no ear issues, daily rhinitis, normal nNO and cilia which appeared normal on a historical HSVM of nasal epithelium (2014). We aim to repeat HSVM on a primary nasal epithelium brushing at the earliest opportunity. We suggest that biallelic mutations in CFAP53 cause congenital situs defects, but may also be responsible for subtle respiratory cilia defects manifesting as adult-onset respiratory problems, although this will require further investigation since the lung disease may have a different aetiology. CFAP53 has not currently been considered in the PCD panel in PanelApp but several other CFAP genes are rated amber in the PCD panel.

The other individual had compound heterozygous stop gain variants in CEP164. nNO was in the normal range, but HSVM analysis showed reduced amplitude of ciliary beating and some long cilia, with $19 \%$ of cilia showing microtubular disorganisation, mostly rearrangements. CEP164 is not recognised as a PCD gene, but has been reported in a case of dual Bardet-Biedl syndrome (BBS) and PCD presentation [40] and our patient had some features of BBS. We are investigating this further as a research case.

\section{Discussion}

Our data show that WGS testing in a clinically wellcharacterised PCD cohort has a high pick-up rate $(16 / 21$ patients received confirmed $(n=9) /$ probable $(\mathrm{n}=4)$ /possible $(\mathrm{n}=3)$ diagnosis $)$ although $12 / 16$ of these could have been picked up by current gene panel testing (Genomic Medicine Service PCD panel (R189), 46 green genes). Most cases were recruited and sequenced as trios (proband and parents), which was generally not required, as a diagnosis could have been achieved by singleton analysis with targeted sequencing of parents to determine phase in a small subset of cases. This suggests that WGS was useful but not costeffective in 13/16 cases. WGS was chosen over WES in 


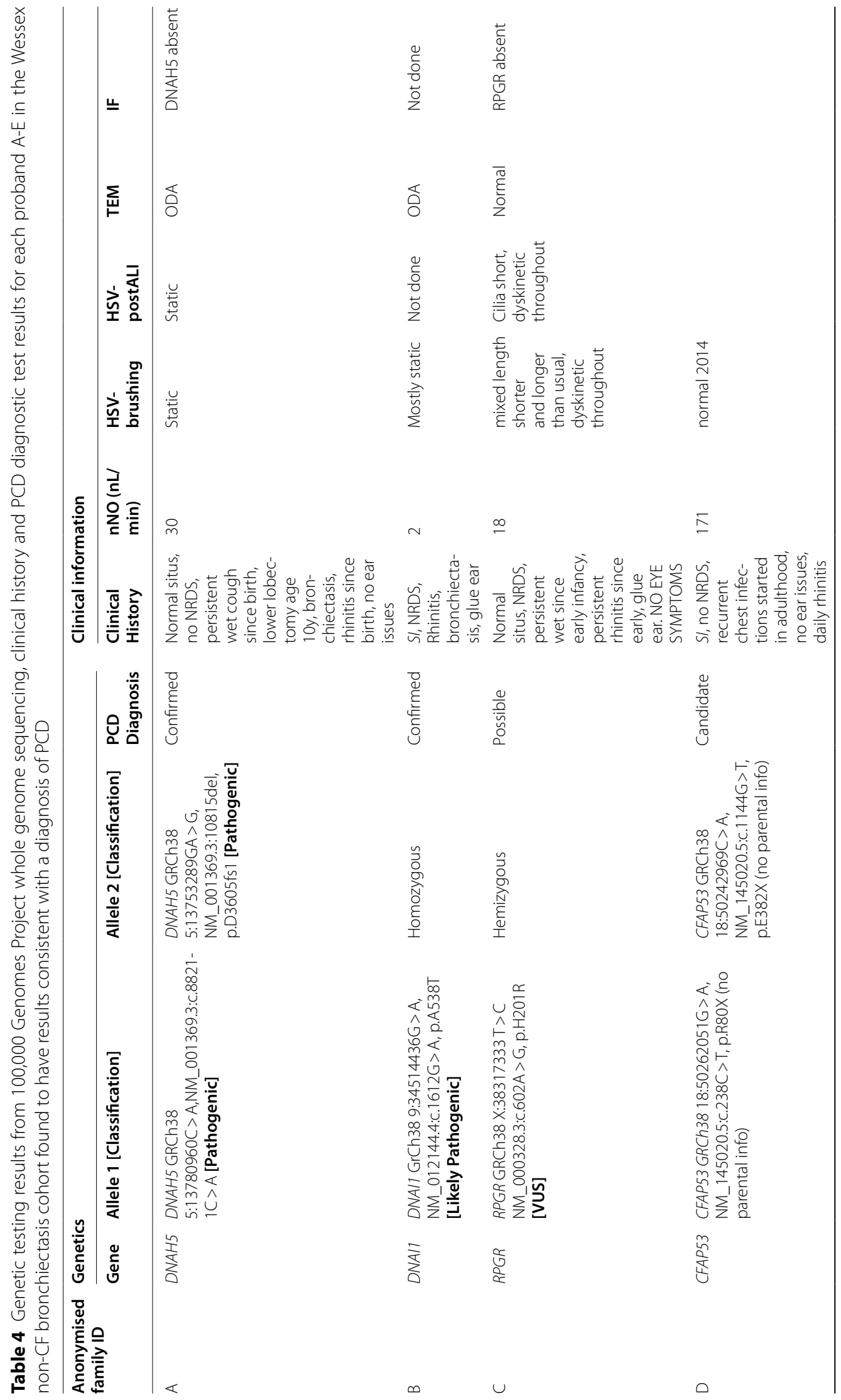




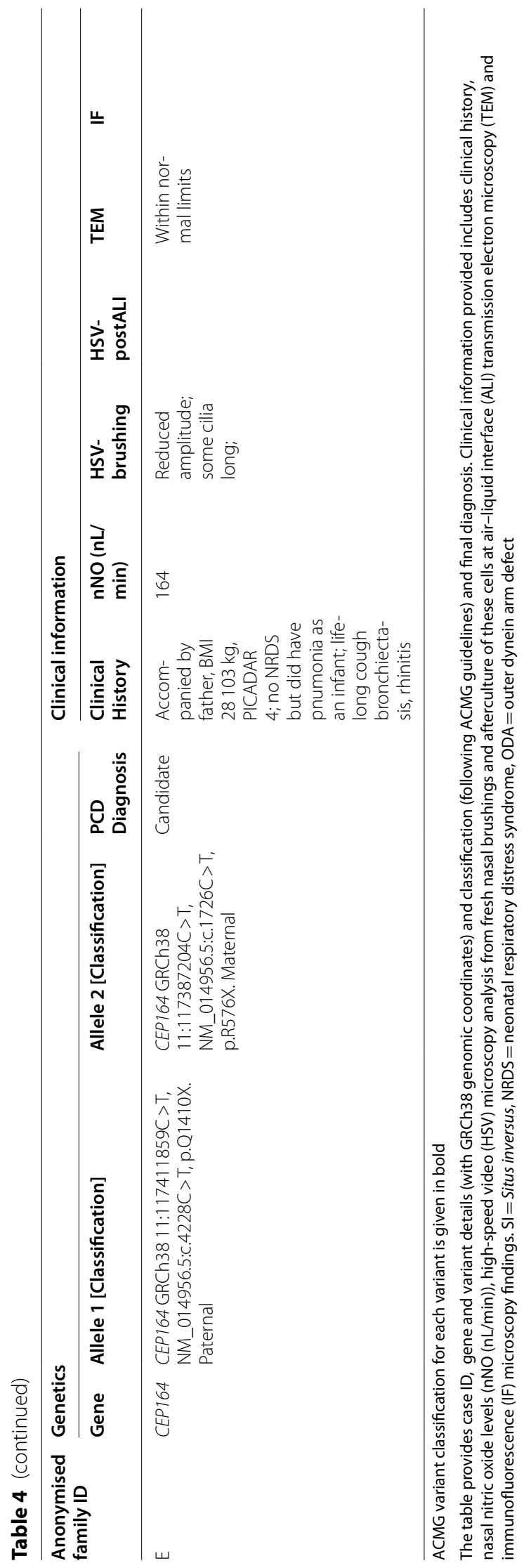




\section{Non-CF bronchiectasis cohort}

$\mathrm{n}=52$ probands

GEL analysis

A: SNVs in non-CF bronchiectasis or primary ciliary gene panel genes (tier $1 / 2$ variants) $\mathrm{B}: \mathrm{SVs}>10 \mathrm{~kb}$ in primary ciliary gene panel genes (tier A variants) (applied to 8 families only)

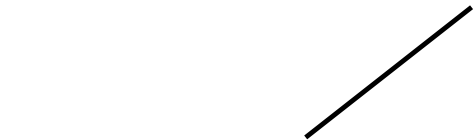

49/52 probands unresolved

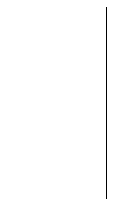

Research analysis

A: biallelic SNVs in cilia candidate genes (from tier 3 variants)

B. monoallelic SNVs in primary ciliary gene panel genes (untiered)

C: SVs $<10 \mathrm{~kb}$ in primary ciliary gene panel genes (untiered)

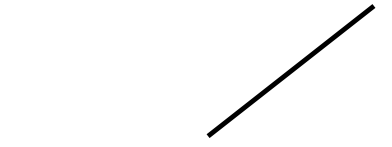

2/49 probands candidate genes

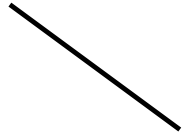

47/49 probands no evidence of PCD
$3 / 52$ probands diagnosed

1 compound heterozygous SNVs

1 homozygous SNV

1 hemizygous SNV

Fig. 5 Flowchart showing clinical and research analyses carried out and all results for Wessex non-CF bronchiectasis cohort

the 100,000 Genomes Project in an attempt to identify which conditions and cases most benefit from WGS over WES in clinical diagnostics, and to provide a large dataset of WGS data for future research. Our study suggests that in the case of PCD, WGS has little advantage over gene panel testing, except in unresolved cases where gene panel testing has not provided genetic diagnoses. In our opinion, gene panel testing is a more sensible first line of testing, followed by WES in unresolved cases. The impact of the 100,000 Genomes Project for PCD testing is that it confirms the efficiency and cost effectiveness of gene panel testing. WGS will unlikely become a frontline diagnostic test for PCD, even in the most well-resourced healthcare systems, but remains an extremely valuable research tool.
In 4/16 diagnoses WGS provided results which would have been missed by gene panel testing, through detection of SVs in trans with pathogenic/likely pathogenic coding SNVs. In some cases these coding SNVs were detected by prior gene panel testing but SV/intronic variants were not detected. 3 of these 4 diagnoses in the PCD cohort were obtained through research analysis of unsolved patients involving extraction of heterozygous SNVs followed by analysis of intronic variants and SVs. The current Genomics England Ltd clinical analysis pipeline does not tier deep intronic SNVs, nor SVs $<10 \mathrm{~kb}$, and is not well optimised to detect cases of SNVs and SVs in trans. Heterozygous SNVs are not tiered in recessive disease, and $\mathrm{SVs}<10 \mathrm{~kb}$ are not tiered by Genomics England Ltd yet 3 of the 4 diagnostic SVs that we found 


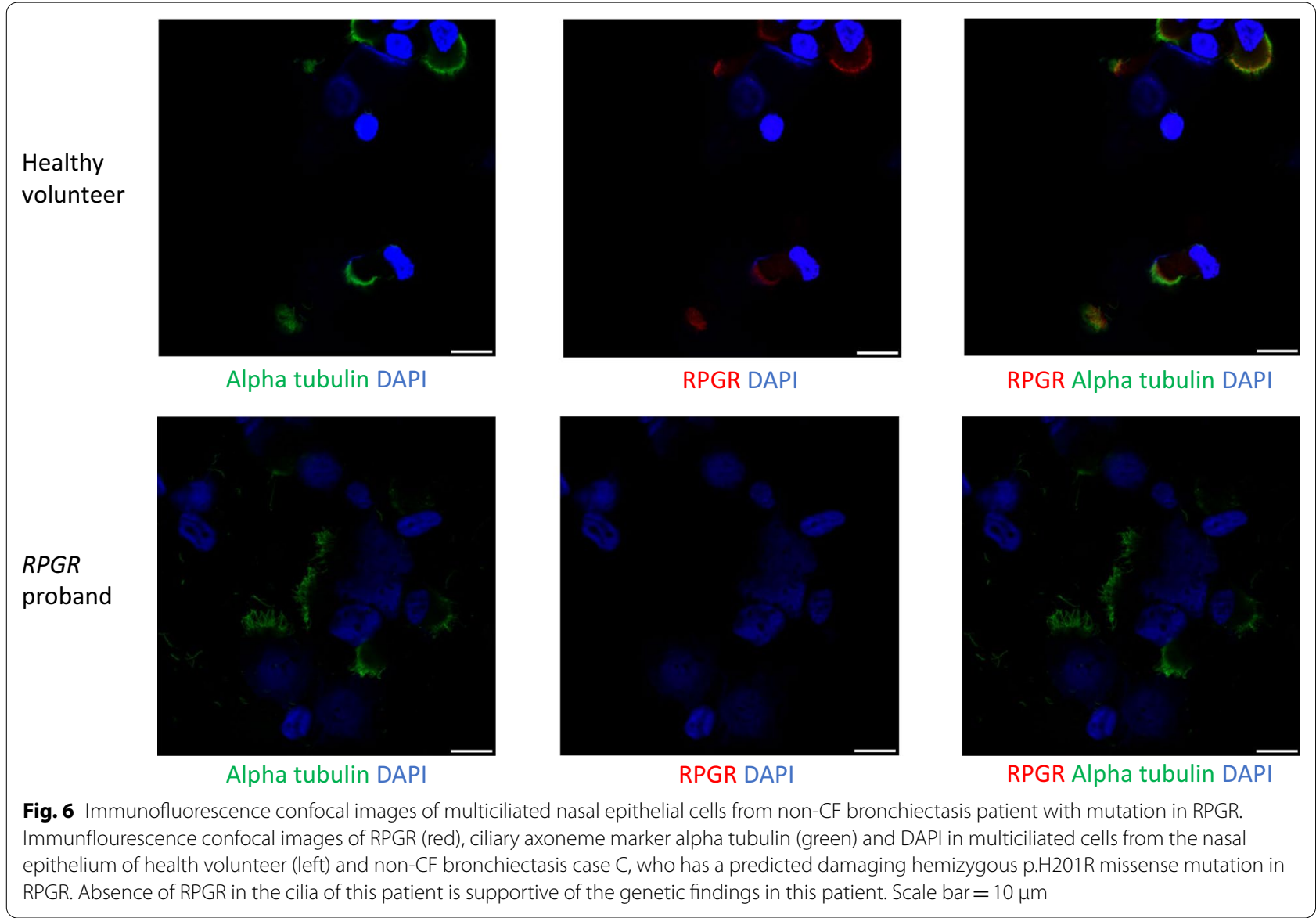

were $<10 \mathrm{~kb}$. The amount of variant data generated by WGS requires filtering, but this needs to be done very carefully to avoid losing relevant variants. Our findings suggest that heterozygous SNVs should be tiered to maximise diagnostic return in clinically well-defined recessive diseases with established genotype/phenotype correlations such as PCD, and SVs should be tiered alongside SNVs in the Genomics England Ltd clinical analysis pipeline.

The identification of a heterozygous CCDC114 splice donor site variant alongside a novel deep intronic VUS highlights that we are likely missing diagnoses by continuing to focus on coding regions in our variant analyses despite sequencing the whole genome. In order to more meaningfully study non-coding regions and synonymous changes we need better tools to predict the effect of such changes on splicing, and allow effective filtering of these. Unless intronic sequences can be screened efficiently and accurately, then WGS may have little advantage over WES (beyond permitting more accurate SV analysis). SpliceAI is a tool with higher sensitivity (0.8987) and specificity (0.9162) for predicting the effect of genetic variants on splicing than any previous tool, but still has false positive and false negative rates of around 10\% [41]. We need to integrate RNA analyses into diagnostics to effectively pick up pathogenic splice variants, but this is difficult in a disease such as PCD in which the affected genes are only expressed in a few tissues of the body, which can't always be easily sampled.

Two of the patients who received a 'confirmed diagnosis' of PCD based on SNVs in known PCD genes had previously received 'probable diagnosis' on the basis of the same variants found in prior gene panel testing. This reclassification of probable to confirmed diagnosis could have been achieved independently with re-review of existing data and targeted gene sequencing in parents. In cases where two variants in a known disease gene have been identified but one or both are VUS re-review of panel/WES data is more useful than further sequencing by WGS, especially if the VUS is in combination with a pathogenic/likely pathogenic variant and phenotype data support the gene of interest. It is clear that re-review of prior testing results is useful to improve diagnoses, and this process would greatly benefit from a motile cilia disease-specific public resource for depositing clinical variant data from PCD patients, similar to CFTR2 (https:// 
cftr2.org/), a public repository for reporting CFTR variants found in CF patients. The BEATPCD consortium is currently working on such a resource, called 'CilioVar' (https://beat-pcd.squarespace.com/working-groupssummary). As a frontline test, WGS provides much greater scope for generating new diagnoses than WES or gene panel testing, as iterative re-analysis would include newly identified disease genes. However, WGS reanalysis brings resource (cost, person hours) implications for storing and analysing large volumes of data. Furthermore, the efficient use of resources also needs to be considered to balance analytical work with diagnostic yield. Although the standardised Genomics England Ltd WGS data analysis pipeline has made significant steps towards streamlining and automating the process of prioritisation of variants likely to be clinically significant, the requirement to carry out expert clinical variant classification to confirm diagnoses remains the rate-limiting step for the implementation of large-scale WGS. Unfortunately, this was poorly resourced in the 100,000 Genomes Project, leading to delays in return of results. It is clear from the 100,000 Genomes Project that adequate funding of data analysis and interpretation is a critical and indispensable component for delivering a WGS-based diagnostic service.

One of the major advantages of WGS over gene panel testing is that it provides significant opportunities for research and novel disease gene discovery. In our study WGS was especially useful in PCD variant negative cases allowing potentially pathogenic variants in candidate genes to be identified in four cases, although these remain to be functionally investigated and proven to be pathogenic. However, for conditions with defined phenotypes and high pickup rates like PCD, fixed panels still have diagnostic utility, so that the more expensive WGS can be reserved for more heterogeneous conditions such as intellectual disability (>1000 known genes) where gene agnostic approaches can be used.

Finally, WGS was useful in helping to confirm that $2 / 21$ patients in the PCD cohort had no genetic evidence to support a diagnosis of PCD. Negative genetic findings prompted these 2 patients to be re-reviewed clinically, suggesting that they are actually unlikely to have PCD. This highlights how difficult PCD can be to diagnose, even in specialist PCD diagnostic centres. The $D R C 1$ case we present demonstrates the important role that genetic testing plays as an additional diagnostic test in cases of PCD where patients do not have a strong history (for example, low PICADAR score [34] and normal nNO). The DCR1 patient was aged 34 years at the time of referral for diagnostic testing, had a PICADAR score of $4 / 14$ which is below the level recommended for referral for diagnostic testing and, unlike many with $D R C 1$ mutations, had normal nitric oxide levels. They therefore risked not proceeding to further diagnostic testing and even following referral, PCD can erroneously be discounted if subtle defects in ciliary beat pattern and TEM are not detected. Cilia structure may appear grossly normal under TEM [13], but further inspection often shows central apparatus abnormalities or microtubule disorganization in a proportion of cilia [17]. The diagnosis in this individual demonstrates the value and importance of careful high-speed video imaging analysis and TEM analysis in suspected cases of PCD, and how genetic testing can help to confirm this analysis. Although this genetic diagnosis could have been identified through gene panel testing and WGS was useful but not essential, we wished to highlight this difficult diagnostic case, and how a diagnosis was obtained.

Alongside 16 genetic diagnoses in the PCD cohort, and identification of candidate genes in 2 additional patients in the PCD cohort, we also obtained genetic diagnosis of $\mathrm{PCD}$ in 3 patients in the non-CF bronchiectasis cohort and identified candidate motile ciliopathy genes in 2 additional patients in this cohort. In contrast to the PCD cohort, the non-CF bronchiectasis cohort comprised adult patients, generally recruited as singletons. Whilst this would not have posed an issue in the PCD cohort, in non-CF bronchiectasis, which is a genetically heterogenous group of disorders, with different patterns of inheritance and some likely nongenetic causes, the difficulty in segregating variants without family member's sequence, and lack of clarity of the pattern of inheritance made analysis more difficult. It would be advantageous to recruit these patients for genetic testing with family members, where possible. These findings show that even in large adult bronchiectasis clinics, the diagnosis of PCD may be overlooked (entered in as bronchiectasis rather than PCD). Therefore, there are likely to be many patients with PCD in adult pulmonology clinics- if clinicians do not revisit the early life history, they may miss patients with a history suggesting a genetic cause. The identification of candidate gene variants in 2 patients in the non-CF bronchiectasis cohort suggests that novel disease genes may be discovered by investigating cases with atypical PCD and that the phenotypic spectrum of PCD may be broader than previously described.

Finally, we present a case of PCD without ophthalmological involvement, associated with a hemizygous variant in RPGR case to provide further evidence that this gene can be a cause of PCD. To be rated green on PanelApp pathogenic variants need to be identified in $\geq 3$ independent families, so identification and publication of additional novel cases in rare/amber genes is important. 


\section{Conclusion}

PCD is a difficult condition to diagnose as there is no "gold standard" diagnostic tool. Genetic testing is useful to confirm a diagnosis of PCD, especially in cases which do not have a typical history, such as in the $D R C 1$ case we present.

PCD diagnoses continue to be missed even in large adult bronchiectasis centres. WGS in adults with non-CF bronchiectasis is useful for detecting missed diagnoses of PCD in this group of patients, and trio analysis is useful in this cohort.

WGS is a useful diagnostic test in a PCD cohort but is only an effective use of resources where patients have received no findings or have only had heterozygous variants found from prior gene panel testing. A significant proportion of diagnoses were missed through the automated Genomics England Ltd tiering pipeline, and were only picked up with additional labour-intensive manual analysis of patient's genome data.

Proband-only gene panel/WGS testing followed by targeted testing of parents where required is sufficient for diagnosis of PCD in most cases, and is more cost-effective.

Heterozygous SNVs and SVs from WGS should not be filtered out of analysis in clinically well-defined recessive diseases with established genotype/phenotype correlations such as PCD.

WGS is a powerful tool for gene discovery. We identify 2 novel candidate PCD genes in our PCD cohort and another 2 in our non-CF bronchiectasis cohort.

The advantages of WGS over gene panel testing will be more apparent when we have better tools for intronic variant filtering and automated systems for clinical variant classification and periodic re-review of data.

\begin{abstract}
Abbreviations
ACMG: American College of Medical Genetics; ALI: Air-liquid interface; AMP: Association for Molecular Pathology; CF: Cystic fibrosis; GMC: Genome Medicine Centre; HSVM: High speed video microscopy; IGV: Integrative Genomics Viewer; MTD: Microtubular disorganisation; N-DRC: Nexin-dynein regulatory complex; nNO: Nasal nitric oxide; NRDS: Neonatal respiratory distress syndrome; PCD: Primary ciliary dyskinesia; SNV: Small nucleotide variant; SV: Structural variant; TEM: Transmission electron microscopy; VUS: Variant of uncertain clinical significance; WES: Whole exome sequencing; WGS: Whole genome sequencing.
\end{abstract}

\section{Supplementary Information}

The online version contains supplementary material available at https://doi. org/10.1186/s12920-021-01084-w.

Additional file 1. Table 1: Genes on 19 and 29 gene next generation sequencing panel used for PCD genetic testing in patients in the Wessex PCD cohort prior to WGS through the 100,000 Genomes Project

Additional file 2. Additional information on 100,000 Genomes Project recruitment, sequencing, sequence analysis and variant filtering and tiering performed by Genomics England Ltd.

\section{Acknowledgements}

We thank the patients and their families for taking part in this research for diagnostics. The National PCD Centre in Southampton is commissioned and funded by NHS England; PCD research is supported by NIHR Southampton Biomedical Research Centre, NIHR Clinical Research Facility, National Institute for Health Research (RFPB PB-PG-1215-20014; and 200470) and The AAIR Charity (Reg. No. 1129698). SNV and SV confirmation was carried out by Dave Bunyan (Wessex Regional Genetics Laboratory). This research was made possible through access to the data and findings generated by the 100,000 Genomes Project. The 100,000 Genomes Project is managed by Genomics England Limited (a wholly owned company of the Department of Health and Social Care). The 100,000 Genomes Project uses data provided by patients and collected by the National Health Service as part of their care and support.

\section{Authors' contributions}

GW conceived of the study, carried out genomic research analysis, and made the figures. The GERC developed the Genomics England pipeline for sequence alignment, variant calling, variant filtering and prioritisation of variants. NST and $\mathrm{DH}$ carried out clinical variant analysis. JL carried out research analysis of variants. GW, NST and JSL wrote the paper. GW, NST, DH, CJ, AH, WTW and JSL contributed to all genetic diagnoses as part of a multidisciplinary team meeting. JC, JT, CL, RD, PG, VM, WTW and JSL contributed to PCD diagnostic decisions as part of a multidisciplinary team meeting. JC carried out IF. JT, JC and CLJ carried out HSVM. RD, PG and JT carried out TEM. AH, BG, MC, WTW and $J S L$ recruited and consented patients and clinically phenotyped participants. All authors approved the submitted version of the manuscript and agreed to be personally accountable for their contributions and the accuracy and integrity of the work. All authors read and approved the final manuscript.

\section{Funding}

GW acknowledges funding from Wellcome Trust (award number 204378/Z/16/Z). The National PCD Centre in Southampton is commissioned and funded by NHS England; PCD research is supported by NIHR Southampton Biomedical Research Centre, NIHR Clinical Research Facility, National Institute for Health Research (RfPB PB-PG-1215-20014; and 200470) and The AAIR Charity (Reg. No. 1129698). JL is supported by an NIHR Research Professorship awarded to Prof Diana Baralle (DB NIHR RP-2016-07-011). The funding bodies played no role in the design of the study and collection, analysis, and interpretation of data and in writing the manuscript.

\section{Availability of data and materials}

The data that support the findings of this study are available from Genomics England Ltd but restrictions apply to the availability of these data, which were used under license for the current study, and so are not publicly available. For further details contact gecip-help@genomicsengland.co.uk. For any other queries contact g.wheway@soton.ac.uk or jlucas1@soton.ac.uk.

\section{Declarations}

\section{Ethics approval and consent to participate}

Written informed consent was obtained from all participants (or parent/ legal guardian) in the 100,000 Genomes Project (IRAS ID 166046; REC reference 14/ EE/1112). GW was granted access to the 100,000 Genomes Project research environment by the Genomics England Access Review Committee, as part of the Hearing and Sight, Neurology and Paediatrics Genomics England Clinical Interpretation Partnerships (GeCIPs). Within the 100,000 Genomes Project research environment, research project RR185'Study of cilia and ciliopathy genes across the 100,000 GP cohort' was registered by GW and approved by Genomics England. This research study received ethical approval from University of Southampton Faculty of Medicine Ethics Committee (ERGO\#54400). Further written informed consent was obtained from all participants (or parent/ legal guardian) recruited for PCD diagnostic testing (National Research Ethics Service South Central (A) Committee 07/Q1702/109) and University of Southampton Faculty of Medicine Ethics Committee ERGO \#53155.

\section{Consent to publication}

Written informed consent was obtained from all participants, or their parents if $<18$ years of age, recruited to the 100,000 Genomes Project for data and clinical details to be used for research and publication as part of the informed consent process (IRAS ID 166046; REC reference 14/EE/1112). 


\section{Competing interests}

JSL reports grants, personal fees and non-financial support from Circassia. GW is employed by Illumina Inc. Other authors declare no competing interests.

\section{Author details}

${ }^{1}$ School of Human Development and Health, Faculty of Medicine, University of Southampton, Southampton, UK. ${ }^{2}$ Institute for Life Sciences, University of Southampton, Southampton, UK. ${ }^{3}$ Wessex Regional Genetics Laboratory, Salisbury NSF Foundation Trust, Salisbury District Hospital, Salisbury, UK. ${ }^{4}$ Wessex Clinical Genetics Service, University Hospitals Southampton NHS Foundation Trust, Southampton, UK. ${ }^{5}$ School of Clinical and Experimental Sciences, Faculty of Medicine, University of Southampton, Southampton, UK. ${ }^{6}$ Primary Ciliary Dyskinesia Centre, NIHR Biomedical Research Centre, University Hospital Southampton NHS Foundation Trust, Southampton, UK. ${ }^{7}$ Biomedical Imaging Unit, University Hospital Southampton NHS Foundation Trust, Southampton, UK. ${ }^{8}$ Queen Alexandra Hospital, Portsmouth Hospitals NHS Trust, Portsmouth, UK.

Received: 24 March 2021 Accepted: 14 September 2021 Published online: 23 September 2021

\section{References}

1. O'Callaghan C, Chetcuti P, Moya E. High prevalence of primary ciliary dyskinesia in a British Asian population. Arch Dis Child. 2010;95(1):51.

2. Kuehni CE, Frischer T, Strippoli MP, Maurer E, Bush A, Nielsen KG, et al. Factors influencing age at diagnosis of primary ciliary dyskinesia in European children. Eur Respir J. 2010;36(6):1248-58.

3. Behan L, Dunn Galvin A, Rubbo B, Masefield S, Copeland F, Manion M, et al. Diagnosing primary ciliary dyskinesia: an international patient perspective. Eur Respir J. 2016;48(4):1096-107.

4. Lucas JS, Barbato A, Collins SA, Goutaki M, Behan L, Caudri D, et al. European Respiratory Society guidelines for the diagnosis of primary ciliary dyskinesia. Eur Respir J. 2017:49(1)

5. Shapiro AJ, Davis SD, Polineni D, Manion M, Rosenfeld M, Dell SD, et al. Diagnosis of primary ciliary dyskinesia an official american thoracic society clinical practice guideline. Am J Respir Crit Care Med. 2018;197(12):e24-39.

6. Fassad MR, Patel MP, Shoemark A, Cullup T, Hayward J, Dixon M, et al. Clinical utility of NGS diagnosis and disease stratification in a multiethnic primary ciliary dyskinesia cohort. J Med Genet. 2020;57(5):322-30.

7. Lucas JS, Rubbo B, Jackson CL, Hirst RA, Hogg C, O'Callaghan C, et al. Response Chest. 2019:156(5):1033-4

8. Rubbo B, Shoemark A, Jackson CL, Hirst R, Thompson J, Hayes J, et al. Accuracy of high-speed video analysis to diagnose primary ciliary dyskinesia. Chest. 2019;155(5):1008-17.

9. Shoemark A, Rubbo B, Legendre M, Fassad MR, Haarman EG, Best S, et al. Topological data analysis reveals genotype-phenotype relationships in primary ciliary dyskinesia. Eur Respir J. 2021.

10. Lucas JS, Davis SD, Omran H, Shoemark A. Primary ciliary dyskinesia in the genomics age. Lancet Respir Med. 2019.

11. Contarini M, Shoemark A, Rademacher J, Finch S, Gramegna A, Gaffuri M, et al. Why, when and how to investigate primary ciliary dyskinesia in adult patients with bronchiectasis. Multidiscip Respiratory Med. 2018;13(Suppl 1):26

12. Turnbull $C$, Scott RH, Thomas $E$, Jones $L$, Murugaesu N, Pretty FB, et al. The 100000 Genomes Project: bringing whole genome sequencing to the NHS. BMJ. 2018;361.

13. Wheway G, Mitchison HM. Opportunities and challenges for molecular understanding of ciliopathies-The 100,000 genomes project. Front Genet. 2019;10:127.

14. Richards S, Aziz N, Bale S, Bick D, Das S, Gastier-Foster J, et al. Standards and guidelines for the interpretation of sequence variants: a joint consensus recommendation of the American College of Medical Genetics and Genomics and the Association for Molecular Pathology. Genet Med. 2015;17(5):405-24.

15. Ellard S, Baple EL, Callaway A, Berry I, Forrester N, Turnbull C, et al. ACGS Best Practice Guidelines for Variant Classification in Rare Disease 2020 https://www.acgs.uk.com/quality/best-practice-guidelines/\#VariantGui delines 2020
16. Jackson CL, Behan L, Collins SA, Goggin PM, Adam EC, Coles JL, et al. Accuracy of diagnostic testing in primary ciliary dyskinesia. Eur Respir J. 2016;47(3):837-48

17. Liu Z, Nguyen QPH, Guan Q, Albulescu A, Erdman L, Mahdaviyeh Y, et al. A quantitative super-resolution imaging toolbox for diagnosis of motile ciliopathies. Sci Transl Med. 2020;12(535).

18. Hirst RA, Jackson CL, Coles JL, Williams G, Rutman A, Goggin PM, et al. Culture of primary ciliary dyskinesia epithelial cells at air-liquid interface can alter ciliary phenotype but remains a robust and informative diagnostic aid. PLOS ONE. 2014;9(2):e89675.

19. Shoemark A, Boon M, Brochhausen C, Bukowy-Bieryllo Z, De Santi MM, Goggin P, et al. International consensus guideline for reporting transmission electron microscopy results in the diagnosis of primary ciliary dyskinesia (BEAT PCD TEM Criteria). Eur Respir J. 2020;55(4).

20. Coles JL, Thompson J, Horton KL, Hirst RA, Griffin P, Williams GM, et al. A revised protocol for culture of airway epithelial cells as a diagnostic tool for primary ciliary dyskinesia. J Clin Med. 2020;9(11).

21. Shoemark A, Frost E, Dixon M, Ollosson S, Kilpin K, Patel M, et al. Accuracy of immunofluorescence in the diagnosis of primary ciliary dyskinesia. Am J Respir Crit Care Med. 2017;196(1):94-101.

22. Martin AR, Williams E, Foulger RE, Leigh S, Daugherty LC, Niblock O, et al. PanelApp crowdsources expert knowledge to establish consensus diagnostic gene panels. Nat Genet. 2019;51(11):1560-5.

23. van Dam TJ, Wheway G, Slaats GG, Group SS, Huynen MA, Giles RH. The SYSCILIA gold standard (SCGSV1) of known ciliary components and its applications within a systems biology consortium. Cilia. 2013:2(1):7-2530-2-7.

24. van Dam TJP, Kennedy J, van der Lee R, de Vrieze E, Wunderlich KA, Rix $\mathrm{S}$, et al. CiliaCarta: an integrated and validated compendium of ciliary genes. PLOS ONE. 2019;14(5):e0216705.

25. Cindrić S, Dougherty GW, Olbrich H, Hjeij R, Loges NT, Amirav I, et al. SPEF2- and HYDIN-mutant cilia lack the central pair-associated protein SPEF2, aiding primary ciliary dyskinesia diagnostics. Am J Respir Cell Mol Biol. 2020;62(3):382-96.

26. Jaganathan K, Kyriazopoulou Panagiotopoulou S, McRae JF, Darbandi SF, Knowles D, Li Yl, et al. Predicting splicing from primary sequence with deep learning. Cell. 2019;176(3):535-48.e24.

27. Hull S, Owen N, Islam F, Tracey-White D, Plagnol V, Holder GE, et al. Nonsyndromic retinal dystrophy due to bi-allelic mutations in the ciliary transport gene IFT140. Invest Ophthalmol Vis Sci. 2016;57(3):1053-62.

28. Xu M, Yang L, Wang F, Li H, Wang X, Wang W, et al. Mutations in human IFT140 cause non-syndromic retinal degeneration. Hum Genet. 2015;134(10):1069-78.

29. Bifari IN, Elkhamary SM, Bolz HJ, Khan AO. The ophthalmic phenotype of IFT140-related ciliopathy ranges from isolated to syndromic congenital retinal dystrophy. Br J Ophthalmol. 2016;100(6):829-33.

30. Pena-Padilla C, Marshall CR, Walker S, Scherer SW, Tavares-Macias G, RazoJimenez G, et al. Compound heterozygous mutations in the IFT140 gene cause Opitz trigonocephaly $\mathrm{C}$ syndrome in a patient with typical features of a ciliopathy. Clin Genet. 2016.

31. Perrault I, Saunier S, Hanein S, Filhol E, Bizet AA, Collins F, et al. MainzerSaldino syndrome is a ciliopathy caused by IFT140 mutations. Am J Hum Genet. 2012;90(5):864-70.

32. Schmidts M, Frank V, Eisenberger T, al Turki S, Bizet AA, Antony D, et al. Combined NGS approaches identify mutations in the intraflagellar transport gene IFT140 in skeletal ciliopathies with early progressive kidney disease. Hum Mutat. 2013;34(5):714-24.

33. Martin CA, Ahmad I, Klingseisen A, Hussain MS, Bicknell LS, Leitch A, et al. Mutations in PLK4, encoding a master regulator of centriole biogenesis, cause microcephaly, growth failure and retinopathy. Nat Genet. 2014;46(12):1283-92.

34. Behan L, Dimitrov BD, Kuehni CE, Hogg C, Carroll M, Evans HJ, et al. PICADAR: a diagnostic predictive tool for primary ciliary dyskinesia. Eur Respir J. 2016:47(4):1103-12

35. Wirschell M, Olbrich H, Werner C, Tritschler D, Bower R, Sale WS, et al. The nexin-dynein regulatory complex subunit DRC1 is essential for motile cilia function in algae and humans. Nat Genet. 2013;45(3):262-8.

36. Olbrich H, Cremers C, Loges NT, Werner C, Nielsen KG, Marthin JK, et al. Loss-of-function GAS8 mutations cause primary ciliary dyskinesia and disrupt the nexin-dynein regulatory complex. Am J Hum Genet. 2015:97(4):546-54. 
37. Narasimhan V, Hjeij R, Vij S, Loges NT, Wallmeier J, Koerner-Rettberg C, et al. Mutations in CCDC11, which encodes a coiled-coil containing ciliary protein, causes situs inversus due to dysmotility of monocilia in the leftright organizer. Hum Mutat. 2015;36(3):307-18.

38. Perles Z, Cinnamon Y, Ta-Shma A, Shaag A, Einbinder T, Rein AJ, et al. A human laterality disorder associated with recessive CCDC11 mutation. J Med Genet. 2012:49(6):386-90.

39. Ide T, Twan WK, Lu H, Ikawa Y, Lim LX, Henninger N, et al. CFAP53 regulates mammalian cilia-type motility patterns through differential localization and recruitment of axonemal dynein components. PLoS Genet. 2020;16(12):e1009232.
40. Shamseldin HE, Shaheen R, Ewida N, Bubshait DK, Alkuraya H, Almardawi

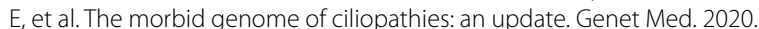

41. Wai HA, Lord J, Lyon M, Gunning A, Kelly H, Cibin P, et al. Blood RNA analysis can increase clinical diagnostic rate andresolve variants of uncertain significance. Genet Med. 2020;22(6):1005-14.

\section{Publisher's Note}

Springer Nature remains neutral with regard to jurisdictional claims in published maps and institutional affiliations.
Ready to submit your research? Choose BMC and benefit from:

- fast, convenient online submission

- thorough peer review by experienced researchers in your field

- rapid publication on acceptance

- support for research data, including large and complex data types

- gold Open Access which fosters wider collaboration and increased citations

- maximum visibility for your research: over 100M website views per year

At BMC, research is always in progress.

Learn more biomedcentral.com/submissions 